\title{
Front Solutions for the Ginzburg-Landau Equation
}

\section{J.-P. Eckmann and Th. Gallay}

Département de Physique Théorique, Université de Genève, CH-1211 Geneva 4, Switzerland

Received June 29, 1992

\begin{abstract}
We prove the existence of front solutions for the Ginzburg-Landau equation

$$
\partial_{t} u(x, t)=\partial_{x}^{2} u(x, t)+\left(1-|u(x, t)|^{2}\right) u(x, t),
$$

interpolating between two stationary solutions of the form $u(x)=\sqrt{1-q^{2}} e^{i q x}$ with different values of $q$ at $x= \pm \infty$. Such fronts are shown to exist when at least one of the $q$ is in the Eckhaus-unstable domain.
\end{abstract}

\section{Introduction}

We consider the Ginzburg-Landau equation (GL)

$$
\partial_{t} u(x, t)=\partial_{x}^{2} u(x, t)+\left(1-|u(x, t)|^{2}\right) u(x, t),
$$

where $u$ is a complex-valued function of $x \in \mathbf{R}$ and $t \in \mathbf{R}_{+}$. This equation has time-independent periodic solutions of the form

$$
u_{q}(x)=\sqrt{1-q^{2}} e^{i \varphi} e^{i q x},
$$

where $q \in[-1,1]$ and $\varphi \in \mathbf{R}$. These stationary solutions are known to be unstable for small amplitudes $\left(q^{2}>1 / 3\right)$ and marginally stable for large amplitudes $\left(q^{2}<1 / 3\right)($ Eckhaus stability, cf. [CE]).

Our aim is to show the existence of front solutions of Eq. (1.1) interpolating between two stationary solutions (1.2). By this, we mean solutions of the form $u(x, t)=U(x, x-c t)$, where $U(x, \xi)$ is a complex function which converges to one of the stationary solutions (1.2), say $u_{q_{0}}(x)$, as $\xi \rightarrow-\infty$ and to another one, say $u_{q_{1}}(x)$, as $\xi \rightarrow+\infty$. Such solutions typically look like a fixed envelope moving to the right with constant velocity $c>0$, while leaving a periodic pattern (the function $\left.u_{q_{0}}\right)$ behind and destroying another one $\left(u_{q_{1}}\right)$ in front, as shown in Fig. 1.

In the case where $u_{q_{1}} \equiv 0\left(q_{1}= \pm 1\right)$, solutions of this form are easily shown to exist, see e.g., [CE, B]. Indeed, inserting in Eq. (1.1) the ansatz $u(x, t)$ 

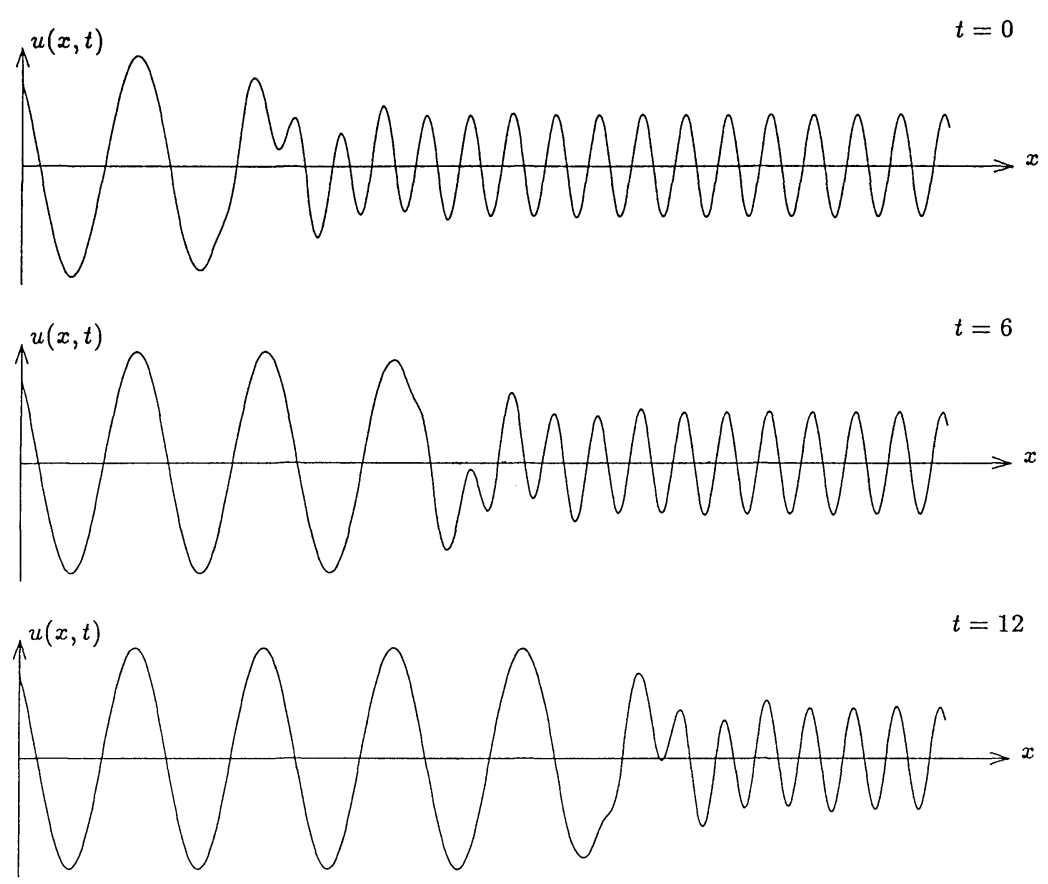

Fig. 1. The real part of a typical front solution of the GL-equation (1.1) is plotted at three different values of $t$. The parameters are $q_{0}=-0.3, q_{1}=0.9$ and $c=5$

$=v(x-c t) e^{i q_{0} x}$, where $v$ is a complex-valued function, $q_{0} \in(-1,1)$ and $c>0$, we obtain an ordinary differential equation for $v$ :

$$
v^{\prime \prime}(\xi)+\left(c+2 i q_{0}\right) v^{\prime}(\xi)+\left(1-q_{0}^{2}-|v(\xi)|^{2}\right) v(\xi)=0 .
$$

Now, defining

$$
H\left(v, v^{\prime}\right)=\frac{1}{2}\left|v^{\prime}\right|^{2}+\frac{1}{2}\left(1-q_{0}^{2}\right)|v|^{2}-\frac{1}{4}|v|^{4},
$$

we can write Eq. (1.3) as a one-dimensional complex Hamiltonian system with Hamiltonian $H$ and (complex) dissipation coefficient $c+2 i q_{0}$; the fixed points are thus given by the local extrema of the "potential" term in Eq. (1.4). It follows that Eq. (1.3) has a stable fixed point $F_{1}$ at $v=0$, and a circle $F_{2}$ of unstable ones $\left(v=\sqrt{1-q_{0}^{2}} e^{i \varphi}\right)$ which corresponds to the stationary solutions (1.2). In view of the "dissipation law" $d H / d \xi=-c\left|v^{\prime}\right|^{2} \leqq 0$, any trajectory entering the region $|v|^{2}<1-q_{0}^{2}, H<\frac{1}{4}\left(1-q_{0}^{2}\right)^{2}$, will stay there and converge to the origin. In particular, $F_{2}$ is on the boundary of this region, and its unstable manifold intersects this region. Therefore, we can conclude the existence of fronts for Eq. (1.1) connecting any solution (1.2) - no matter whether stable or not-to the origin.

The case where both stationary solutions $u_{q_{0}}, u_{q_{1}}$ are non-zero is harder. As a matter of fact, we cannot make the ansatz $u(x, t)=v_{0}(x-c t) e^{i q_{0} x}$ $+v_{1}(x-c t) e^{i q_{1} x}$, for as soon as $u$ contains a superposition of any two different wave-numbers $q_{0}, q_{1}$, the non-linear term $|u|^{2} u$ in Eq. (1.1) produces all the 
"harmonics" $q_{n}=q_{0}+n\left(q_{1}-q_{0}\right), n \in \mathbf{Z}$. So, the simplest expression we can hope for is

$$
u(x, t)=\sum_{n \in \mathbf{Z}} C_{n}(x-c t) e^{i q_{n} x} .
$$

Inserting in Eq. (1.1), we obtain the following system for the $C_{n}$ :

$$
\begin{aligned}
& C_{n}^{\prime}=D_{n}, \\
& D_{n}^{\prime}=-\left(c+2 i q_{n}\right) D_{n}+C_{n}\left(q_{n}^{2}-1\right)+F_{n}(C),
\end{aligned}
$$

where $F=\left(F_{n}\right)_{n \in \mathbf{Z}}$ is the non-linear term

$$
F_{n}(C)=\sum_{p+s+r=n} C_{p} C_{s} C_{-r}^{*}
$$

and the symbol' means the derivative with respect to $\xi=x-c t$.

We are thus looking for solutions $C(\xi)=\left(C_{n}(\xi)\right)_{n \in \mathbf{Z}}$ of Eq. (1.6) subject to the boundary conditions

$$
\lim _{\xi \rightarrow-\infty} C(\xi) \in \mathrm{F}_{2}, \lim _{\xi \rightarrow+\infty} C(\xi) \in \mathrm{F}_{3}
$$

where

$$
\begin{aligned}
& \mathrm{F}_{2}=\left\{\left(C_{n}\right)_{n \in \mathbf{Z}}|| C_{n} \mid=\sqrt{1-q_{0}^{2}} \delta_{n, 0}\right\}, \\
& \mathrm{F}_{3}=\left\{\left(C_{n}\right)_{n \in \mathbf{Z}}|| C_{n} \mid=\sqrt{1-q_{1}^{2}} \delta_{n, 1}\right\},
\end{aligned}
$$

are the circles of fixed points of Eq. (1.6) corresponding to the stationary solutions $u_{q_{0}}, u_{q_{1}}$ respectively. More precisely, the question we are interested in is the following: for which $q_{0}, q_{1} \in[-1,1]$ do solutions of Eqs. (1.6), (1.8) exist? A partial answer is given by the two theorems below, which constitute the main result of this paper.

Theorem 1.1. (Unstable-Unstable case) Let $0<\alpha<1 / 2, c>0$. There exists an $\varepsilon_{1}=\varepsilon_{1}(c)>0$ such that, for every $\varepsilon \leqq \varepsilon_{1}$, there is a solution of Eqs. (1.6), (1.8) with $q_{0}=-1+\varepsilon$ and $q_{1}=1-\alpha \varepsilon$. Moreover, $\varepsilon_{1}(c)$ has a (strictly) positive limit as $c \rightarrow \infty$.

Theorem 1.2. (Stable-Unstable case) Let $-1 / \sqrt{3}<q_{0} \leqq 0$. There exist an $\varepsilon_{1}>0$ and a $c_{1}>0$ such that, for all $\varepsilon \leqq \varepsilon_{1}$ and all $c \geqq c_{1}$, Eqs. (1.6), (1.8) have a solution with $q_{1}=\sqrt{1-\varepsilon^{2}}$.

Remark. The problem of constructing front solutions is phase covariant in the following sense. The system Eq. (1.6) has two continuous symmetries, which reflect the phase and translation invariance of the GL-equation Eq. (1.1). Indeed, defining the transformation $R_{\varphi}$ by $\left(R_{\varphi} C\right)_{n}=e^{i \varphi} C_{n}$, we see from Eq. (1.7) that $F \circ R_{\varphi}=R_{\varphi} F$ for all $\varphi \in[0,2 \pi]$. Similarly, $F$ commutes with $T_{\delta}$, where $\left(T_{\delta} C\right)_{n}=e^{i n \delta} C_{n}$. As a consequence, as soon as any pair of points of $F_{2}$ and $F_{3}$ are connected by an orbit of Eq. (1.6), the same is true for any other pair, since the two operations $R$ and $T$ allow to rotate the circles $\mathrm{F}_{2}, \mathrm{~F}_{3}$ independently.

We shall briefly comment on the range of validity of the theorems (in $q_{0}, q_{1}$ ). First of all, a nice application of the Maximum Principle for parabolic equations shows that, if $u(x, t)$ is any solution of Eq. (1.1), the number of zeros of $\operatorname{Re}(u(x, t))$ is (locally in $x$ ) non-increasing in time [A]. This means that front solutions can only 


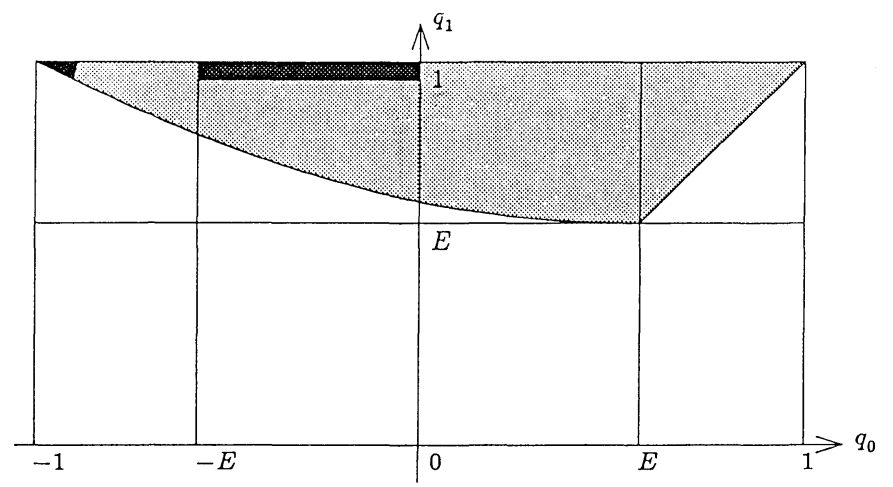

Fig. 2. The shaded region shows the values of $q_{0}, q_{1}$ for which we expect front solutions generically to occur. The black regions are the domains of validity of Theorem 1.1 and Theorem 1.2. The constant $E=1 / \sqrt{3}$ is the threshold of the Eckhaus instability

exist for $\left|q_{1}\right|>\left|q_{0}\right|$, if $c>0$, as is easily seen from Fig. 1. Moreover, since Eq. (1.1) is invariant under the complex conjugation $u \rightarrow u^{*}$, there is no loss in generality in assuming that $q_{1}>\left|q_{0}\right|$. Finally, some genericity considerations which will be explained at the end of Sect. 4.2 lead us to suppose that $\left(q_{0}-q_{1}\right)^{2}<6 q_{1}^{2}-2$. Combining these conditions we obtain the shaded region in Fig. 2.

Now, let us choose $q_{0}, q_{1}$ in this shaded region and consider the sequence of wave-numbers $q_{n}=q_{0}+n\left(q_{1}-q_{0}\right)$. If the difference $q_{1}-q_{0}$ is sufficiently small, many $q_{n}$ lie in the interval $[-1,1]$ and, by Eq. (1.2), there corresponds to each of them a stationary solution $u_{q_{n}}$. Thus, as well as between $u_{q_{0}}$ and $u_{q_{1}}$, one can imagine fronts between $u_{q-1}$ and $u_{q_{1}}, u_{q_{-2}}$ and $u_{q_{1}}, \ldots$, all of them being solutions of the same system Eq. (1.6) with different boundary conditions (1.8). So, to avoid inessential complications, we restrict ourselves to the case $q_{0} \leqq 0$ in which there is only one possibility of constructing a front solution, namely between $u_{q_{0}}$ and $u_{q_{1}}$. In this situation, we expect this solution to exist for all $q_{0}, q_{1}$ in the shaded region, and this is well confirmed by numerical simulations. However, the domain in which we prove it (Theorem 1.1, Theorem 1.2) is much smaller: it is the black region in Fig. 2.

\section{Preliminaries}

We begin our analysis of the dynamical system Eq. (1.6) by diagonalizing the linear part of the right-hand side. The corresponding operator is already block diagonal with $2 \times 2$ blocks labelled by $n \in \mathbf{Z}$; the $n^{\text {th }}$ block is just the linear part of the equation for $\left(C_{n}, D_{n}\right)$, and its eigenvalues are given by

$$
\lambda_{n \pm}=\frac{1}{2}\left(-\left(c+2 i q_{n}\right) \pm \sqrt{c^{2}-4+4 i c q_{n}}\right) .
$$

So, defining the new variables

$$
\left(\begin{array}{c}
C_{n} \\
D_{n}
\end{array}\right)=\left(\begin{array}{cc}
1 & 1 \\
\lambda_{n+} & \lambda_{n-}
\end{array}\right)\left(\begin{array}{c}
A_{n} \\
B_{n}
\end{array}\right)
$$


we obtain the following system:

$$
\left(\begin{array}{l}
A_{n}^{\prime} \\
B_{n}^{\prime}
\end{array}\right)=\left(\begin{array}{cc}
\lambda_{n+} & 0 \\
0 & \lambda_{n-}
\end{array}\right)\left(\begin{array}{l}
A_{n} \\
B_{n}
\end{array}\right)+\frac{1}{\lambda_{n+}-\lambda_{n-}}\left(\begin{array}{r}
F_{n}(A+B) \\
-F_{n}(A+B)
\end{array}\right),
$$

where $(A+B)_{n} \equiv A_{n}+B_{n}=C_{n}$ by Eq. (2.2). In these variables, the circles of fixed points $\mathrm{F}_{2}, \mathrm{~F}_{3}$ become

$$
\begin{aligned}
& \left.\mathrm{F}_{2}\right) A_{0}=\frac{-\lambda_{0-}}{\lambda_{0+}-\lambda_{0-}} \sqrt{1-q_{0}^{2}} e^{i \varphi}, \quad B_{0}=\frac{\lambda_{0+}}{\lambda_{0+}-\lambda_{0-}} \sqrt{1-q_{0}^{2}} e^{i \varphi}, \quad \varphi \in \mathbf{R}, \\
& \left.\mathrm{F}_{3}\right) A_{1}=\frac{-\lambda_{1-}}{\lambda_{1+}-\lambda_{1}-} \sqrt{1-q_{1}^{2}} e^{i \psi}, \quad B_{1}=\frac{\lambda_{1+}}{\lambda_{1+}-\lambda_{1}-} \sqrt{1-q_{1}^{2}} e^{i \psi}, \quad \psi \in \mathbf{R},
\end{aligned}
$$

all the other $A_{n}, B_{n}$ being equal to zero.

\subsection{The Function Space. Let $(\mathscr{H},(\cdot, \cdot))$ be the Hilbert space}

$$
\mathscr{H}=\left\{\left.\left(A_{n}\right)_{n \in \mathbf{Z}}\left|\sum_{n \in \mathbf{Z}}(1+|n|)^{6}\right| A_{n}\right|^{2}<\infty\right\}, \quad(A, \tilde{A})=\sum_{n \in \mathbf{Z}}(1+|n|)^{6} A_{n}^{*} \tilde{A}_{n},
$$

and denote by $\mathscr{H}^{2}$ the direct sum $\mathscr{H} \oplus \mathscr{H}$. From now on, we mean by a solution of the system a $\mathscr{C}^{1}$ curve $\xi \rightarrow(A(\xi), B(\xi))$ in $\mathscr{H}^{2}$, such that Eq. (2.3) is satisfied. If this is the case, then (by construction) $C_{n}(\xi)=A_{n}(\xi)+B_{n}(\xi)$ is of class $\mathscr{C}^{2}$ for all $n$, and Eq. (1.6) is verified. Moreover, since

$$
\sum_{n \in \mathbf{Z}}(1+|n|)^{2}\left|C_{n}(\xi)\right| \leqq\left(\sum_{n \in \mathbf{Z}} \frac{1}{(1+|n|)^{2}}\right)^{1 / 2}\|C(\xi)\|,
$$

it is easy to see that $u(x, t)$ defined by the sum $(1.5)$ is $\mathscr{C}^{2}$ in $x$ and $t$, and verifies the GL-equation Eq. (1.1).

The space $\mathscr{H}$ is mapped into itself by the non-linear term (1.7). Indeed, a standard result in Sobolev space theory (see e.g., [CE]) says that convolution is a continuous bilinear map from $\mathscr{H} \times \mathscr{H}$ into $\mathscr{H}$. This means that there exists a $K>0$ such that $\|A * B\| \leqq K\|A\|\|B\|$ for all $A, B \in \mathscr{H}$. Now, $F(C)$ is nothing but the double convolution $C * C * \bar{C}$, where ${ }^{-}: \mathscr{H} \rightarrow \mathscr{H}$ is the antilinear isometry defined by $(\bar{C})_{n}=\left(C_{-n}\right)^{*}$. So, $F: \mathscr{H} \rightarrow \mathscr{H}$ is $\mathscr{C}^{\infty}$ and

$$
\|F(C)-F(\tilde{C})\| \leqq 3 K^{2} r^{2}\|C-\tilde{C}\|,
$$

for all $C, \tilde{C} \in \mathscr{H}$ such that $\|C\|,\|\tilde{C}\| \leqq r$. In the sequel, if $\mathscr{E}$ is some normed space and $f: \mathscr{E} \rightarrow \mathscr{E}$ some Lipschitz map, we shall denote by $\mathscr{B}_{r} \subset \mathscr{E}$ the ball of radius $r$ around the origin in $\mathscr{E}$ and by $\operatorname{Lip}(f)$ the Lipschitz constant of $f$. With these notations, Eq. (2.5) simply means that $\operatorname{Lip}(F) \leq 3 K^{2} r^{2}$ in $\mathscr{B}_{r} \subset \mathscr{H}$.

2.2. Spectral Properties and Invariant Manifolds. Figures 3 and 4 show the real part of the spectrum (2.1), plotted as a function of the wave-number $q$. The points where $q=q_{n} \equiv q_{0}+n\left(q_{1}-q_{0}\right)$ for some $n \in \mathbf{Z}$ correspond to the eigenvalues of the system. The two branches ( + and - ) cross at $q=0$ if $c \leq 2$; otherwise, they are separated by a distance growing like $c$ as $c \rightarrow \infty$. In all cases, eigenvalues with zero real part only occur if $q_{n}= \pm 1$ for some $n \in \mathbf{Z}$. 


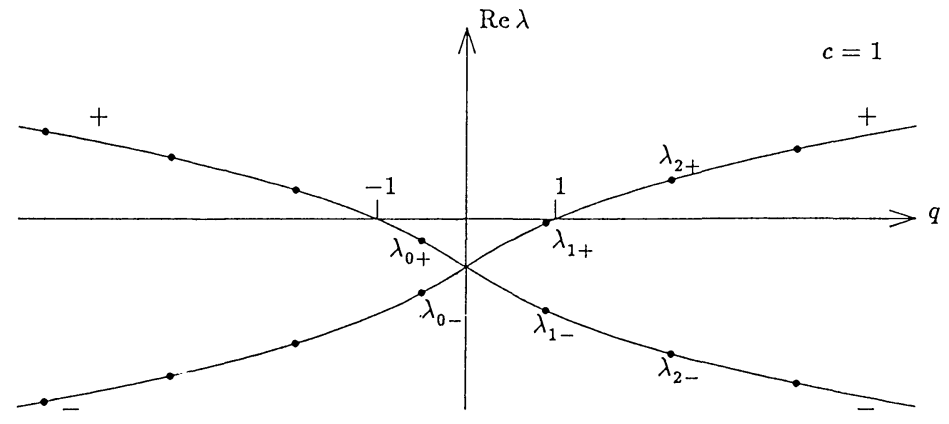

Fig. 3. The real part of the spectrum (2.1), in the case $c<2$

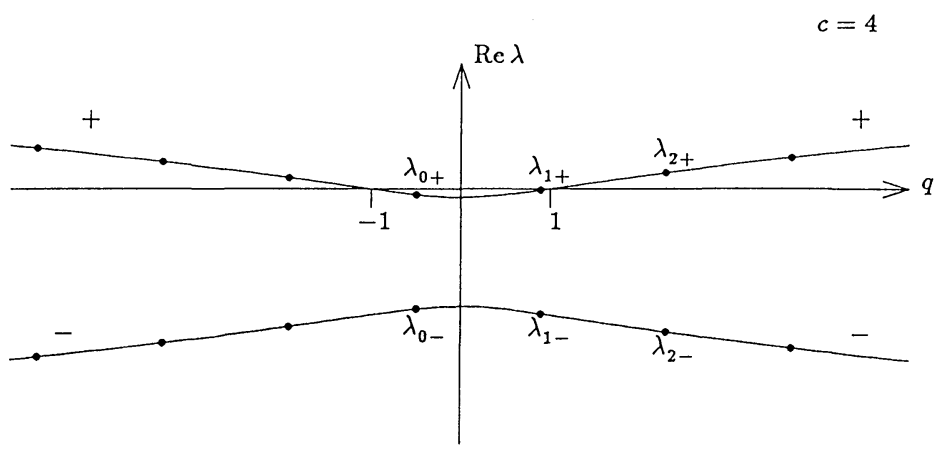

Fig. 4. The real part of the spectrum (2.1), in the case $c>2$

In view of these spectral properties, our strategies for the proofs of Theorem 1.1 and Theorem 1.2 are very natural. In the "unstable-unstable case" (Sect. 3), we fix $\alpha \in(0,1 / 2), c>0$, and define $q_{0}=-1+\varepsilon, q_{1}=1-\alpha \varepsilon$ for some (small) $\varepsilon>0$. The spectrum (2.1) thus contains two "central" eigenvalues (that is, $\operatorname{Re} \lambda_{0+}$ and $\operatorname{Re} \lambda_{1+}$ are $\mathcal{O}(\varepsilon)$ ), while the real parts of all the other ones are bounded away from zero as $\varepsilon \rightarrow 0$. Using this information, we consider the evolution of the system (2.3) on the local invariant manifold corresponding to these two central directions. Applying the general theory reported in Appendix A, we shall prove the existence of such a center manifold in a neighborhood of the origin whose size does not depend on $\varepsilon$. Since the circles $F_{2}, F_{3}$ shrink to zero as $\varepsilon \rightarrow 0$, all these fixed points will belong to the center manifold if $\varepsilon$ is sufficiently small. As a consequence, we shall prove the existence of front solutions connecting $F_{2}$ to $F_{3}$ by simply studying the resulting flow on the center manifold.

In the "stable-unstable case" (Sect. 4), we choose $q_{0}, q_{1}$ such that $q_{0}^{2}<1 / 3$, $1 / \sqrt{3}<q_{1}<1$. We do not follow the same procedure as above, because the fixed point $\mathrm{F}_{2}$ corresponding to $q_{0}$ is no longer close to zero, so that we have no guarantee that it would lie on the local center manifold which we would construct. We rather consider the evolution of the system (2.3) on the (infinite-dimensional) invariant manifold corresponding to the upper branch (labelled " + ") of the spectrum. Using $c$ as a parameter, we shall prove the existence of such a centerunstable manifold in a neighborhood of size $\mathcal{O}(c)$ of the origin, thus containing the 
fixed points $F_{2}, F_{3}$ if $c$ is sufficiently large. We shall then study the resulting semiflow on the manifold, and prove the existence of front solutions.

\section{Proof of the Unstable-Unstable Case}

As indicated, we fix $\alpha \in(0,1 / 2), c>0$, and define $q_{0}=-1+\varepsilon, q_{1}=1-\alpha \varepsilon$ for some small $\varepsilon>0$. To avoid complications, we assume from the outset that $\varepsilon \leqq 1 / 10$.

3.1. Spectral Properties. We first describe in detail the spectrum (2.1) by performing perturbation theory in $\varepsilon$. All calculations are omitted, being straightforward. We find for the two central directions

$$
\lambda_{0+}=-\frac{2 \varepsilon}{c-2 i}+\mathcal{O}\left(\frac{c^{2} \varepsilon^{2}}{(c+2)^{3}}\right), \quad \lambda_{1+}=-\frac{2 \alpha \varepsilon}{c+2 i}+\mathcal{O}\left(\frac{c^{2} \varepsilon^{2}}{(c+2)^{3}}\right),
$$

and for the other eigenvalues

$$
\operatorname{Re} \lambda_{n+} \geqq \frac{c}{(c+2)^{2}}, n \neq 0,1 ; \quad \operatorname{Re} \lambda_{n-}=-c-\operatorname{Re} \lambda_{n+}, n \in \mathbf{Z} .
$$

Moreover, using the identity $\lambda_{n+}-\lambda_{n-}=\sqrt{c^{2}-4+4 i c q_{n}}$, we obtain

$$
\frac{1}{\left|\lambda_{n+}-\lambda_{n-}\right|} \leqq \frac{2}{c+2}, \quad n \in \mathbf{Z} \text {. }
$$

Finally, for $n=0,1$, we also have

$$
\frac{1}{\lambda_{0+}-\lambda_{0-}}=\frac{1}{c-2 i}+\mathcal{O}\left(\frac{c \varepsilon}{(c+2)^{3}}\right), \quad \frac{1}{\lambda_{1+}-\lambda_{1-}}=\frac{1}{c+2 i}+\mathcal{O}\left(\frac{c \varepsilon}{(c+2)^{3}}\right) \text {. }
$$

3.2. Reduction to the Center Manifold. Using the estimates above, we now reduce the system (2.3) to a center manifold corresponding to the eigenvalues $\lambda_{0+}, \lambda_{1+}$. The first main result of this subsection is:

Proposition 3.1. There is a $K_{0}>0$ such that, for all $\varepsilon<K_{0} c /(c+2)$, the system (2.3) defines a flow on a two-dimensional local center manifold of radius $\mathcal{O}\left(\varepsilon^{1 / 2}\right)$, which contains the fixed points $\mathrm{F}_{2}, \mathrm{~F}_{3}$.

Definition. We define $\varepsilon_{c}=K_{0} c /(c+2)$.

Proof. In a first stage, we show the existence of a center-unstable manifold associated with the branch $\left\{\lambda_{n+}\right\}_{n \in \mathbf{Z}}$ of the spectrum, by applying Theorem A.1 to the system obained from Eq. (2.3) by reversing the sign of the "time" $\xi$. Using the notations of Appendix A, we set $\mathscr{E}^{c s}=\left\{\left(A_{n}\right)_{n \in \mathbf{Z}}\right\} \cong \mathscr{H}, \mathscr{E}^{u}=\left\{\left(B_{n}\right)_{n \in \mathbf{Z}}\right\} \cong \mathscr{H}$, and we define the linear operators $A^{c s}, A^{u}$ by $\left(A^{c s} A\right)_{n}=-\lambda_{n+} A_{n},\left(A^{u} B\right)_{n}=-\lambda_{n-} B_{n}$. According to Eqs. (3.1), (3.2), we have $\operatorname{Re}\left(-\lambda_{n+}\right) \leqq \operatorname{Re}\left(-\lambda_{0+}\right) \leqq c /\left(c^{2}+4\right) \leqq c / 4$ for all $n \in \mathbf{Z}$, and $\operatorname{Re}\left(-\lambda_{n_{-}}\right) \geqq 3 c / 4$. Thus, the assumption H1 of Theorem A.1 is satisfied with $\lambda^{c s}=c / 4, \lambda^{u}=3 c / 4$ and $D=1$ (since $A^{c s}, A^{u}$ are diagonal). On the other hand, the non-linear terms in Eq. (2.3) are $\mathscr{C}^{\infty}$, phase covariant, and vanish at the origin together with their derivatives, so that $\mathrm{H} 2$ is satisfied. Moreover, if 
$\ell^{c s}(r), \ell^{u}(r)$ denote their Lipschitz constants in $\mathscr{B}_{r} \subset \mathscr{E}^{c s} \oplus \mathscr{E}^{u}$, it follows from Eqs. (2.5), (3.3) that $\ell^{c s}(r), \ell^{u}(r) \leqq C r^{2} /(c+2)$ for some $C>0$. So, choosing $\beta=c / 3$ and defining $r_{1}^{2} \equiv K_{1} c(c+2)$ for some sufficiently small $K_{1}>0$, we can bound $\sigma(r, \beta)$ in Eq. (A.2) by $\frac{1}{2}\left(r / r_{1}\right)^{2}$, which is smaller than $1 / 2$ if $r<r_{1}$. Applying Theorem A.1, we thus obtain:

Lemma 3.2. There is a $K_{1}>0$ such that, for all $r<r_{1}=\sqrt{K_{1} c(c+2)}$, there exists a phase covariant $\mathscr{C}^{1,1}$ function $h: \mathscr{E}^{c s} \rightarrow \mathscr{E}^{u}$ with $h(0)=0, D h(0)=0$, whose graph (restricted to $\mathscr{B}_{r}$ ) is a local center-unstable manifold for the system (2.3). Moreover, $\operatorname{Lip}(h) \leqq \frac{1}{2}\left(r / r_{1}\right)^{2}$.

Thus, the system (2.3) defines on the center-unstable manifold $B=h(A)$ a semiflow (for $\xi \leqq 0$ ) whose projection onto $\mathscr{E}^{c s}$ verifies the differential equation

$$
A_{n}^{\prime}=\lambda_{n+} A_{n}+\frac{1}{\lambda_{n+}-\lambda_{n-}} F_{n}(A+h(A)), \quad A \in \mathscr{B}_{r} \subset \mathscr{H} .
$$

In a second stage, we reduce the system (3.5) to the two-dimensional centerstable manifold associated with the eigenvalues $\lambda_{0+}, \lambda_{1+}$. Let now $\mathscr{E}^{c s} \equiv \mathscr{H}_{0} \cong \mathbf{C}^{2}$ be the subspace of $\mathscr{H}$ spanned by the two central directions $A_{0}, A_{1}$, and $\mathscr{E}^{u}$ its orthogonal complement in $\mathscr{H}$. Proceeding as above, we define the linear operators $A^{c s}, A^{u}$ by $A^{c s}\left(A_{0}, A_{1}\right)=\left(\lambda_{0+} A_{0}, \lambda_{1} A_{1}\right)$ and $\left(A^{u} A\right)_{n}=\lambda_{n+} A_{n}, n \neq 0,1$. In view of Eqs. (3.1), (3.2), the assumption H1 of Theorem A.1 is verified if we take $\lambda^{c s}=0, \lambda^{u}=c /(c+2)^{2}$, and $D=1$. On the other hand, denoting by $\ell^{c s}(r), \ell^{u}(r)$ the Lipschitz constants in $\mathscr{B}_{r} \subset \mathscr{H}$ of the non-linear terms in Eq. (3.5), we see from Eqs. (2.5), (3.3) and Lemma 3.2 that $\mathrm{H} 2$ is satisfied, and that $\ell^{c s}(r), \ell^{u}(r) \leqq C r^{2} /(c+2)$ for some $C>0$. So, choosing $\beta=\lambda^{u} / 3$ and defining $r_{2}^{2}=K_{2} c /(c+2)$ for some sufficiently small $K_{2} \leqq K_{1}$, we have $\sigma(r, \beta) \leqq \frac{1}{2}\left(r / r_{2}\right)^{2}$ in Eq. (A.2). Applying Theorem A.1, we obtain:

Lemma 3.3. There is a $K_{2}>0$ such that, for all $r<r_{2}=\sqrt{K_{2} c /(c+2)}$, there exists a phase covariant $\mathscr{C}^{1,1}$ function $g: \mathscr{E}^{c s} \rightarrow \mathscr{E}^{u}$ with $g(0)=0, D g(0)=0$, whose graph (restricted to $\mathscr{B}_{r}$ ) is a local center-stable manifold for the system (3.5). Moreover, $\operatorname{Lip}(g) \leqq \frac{1}{2}\left(r / r_{2}\right)^{2}$.

Combining the two lemmas we obtain the existence, if $r<r_{2}$, of the local center manifold $\Gamma_{r}=\left\{(A, B) \in \mathscr{H}^{2} \mid A=(a, g(a)), B=h(A), a \in \mathscr{B}_{r} \subset \mathscr{H}_{0}\right\}$. Furthermore, the projection onto $\mathscr{H}_{0}$ of the flow defined on $\Gamma_{r}$ by Eq. (2.3) verifies the differential equation

$$
\begin{aligned}
& A_{0}^{\prime}=\lambda_{0+} A_{0}+\frac{1}{\lambda_{0+}-\lambda_{0-}} F_{0}(a+k(a)), \\
& A_{1}^{\prime}=\lambda_{1+} A_{1}+\frac{1}{\lambda_{1+}-\lambda_{1-}} F_{1}(a+k(a)),
\end{aligned}
$$

where $a=\left(A_{0}, A_{1}\right) \in \mathscr{B}_{r} \subset \mathscr{H}_{0}$ and $k: \mathscr{H}_{0} \rightarrow \mathscr{H}$ is defined by the identity $(a, g(a))+h(a, g(a))=a+k(a)$. So, $k$ is phase covariant, $k(0)=0, D k(0)=0$, and $\operatorname{Lip}(k) \leqq \ell\left(r / r_{2}\right)^{2}$ for some $\ell>0$.

We now complete the proof of Proposition 3.1. Consider the fixed points (2.4). Using Eqs. (3.1), (3.3) and recalling that $\sqrt{1-q_{1}^{2}}, \sqrt{1-q_{0}^{2}}$ are $\mathcal{O}\left(\varepsilon^{1 / 2}\right)$, we see that there exists an $R>0$ such that $\mathrm{F}_{2}, \mathrm{~F}_{3} \in \mathscr{B}_{R \varepsilon^{1 / 2}} \subset \mathscr{H}^{2}$ for all $\varepsilon>0$. Thus, if 
$R \varepsilon^{1 / 2}<r_{2}$, these fixed points will lie on the center manifold $\Gamma_{R \varepsilon^{1 / 2}}$ (see Remark 3 after Theorem A.1). Thus, defining $\varepsilon_{c}=\left(r_{2} / R\right)^{2}=K_{0} c /(c+2)$ and noting that $\left(R \varepsilon^{1 / 2} / r_{2}\right)^{2} \equiv \varepsilon / \varepsilon_{c}$, the proof of Proposition 3.1 is complete.

The proof gives us the further result:

Corollary 3.4. The projection onto $\mathscr{H}_{0} \cong \mathbf{C}^{2}$ of the flow defined on the center manifold by Eq. (2.3) verifies the differential equation Eq. (3.6) with $k: \mathscr{H}_{0} \rightarrow \mathscr{H}$ a phase covariant $\mathscr{C}^{1,1}$ function verifying $k(0)=0, D k(0)=0, \operatorname{Lip}(k) \leqq \ell \varepsilon / \varepsilon_{c}$.

This result reduces the proof of Theorem 1.1 to the study of the system (3.6) in the ball $\mathscr{B}_{R \varepsilon^{1 / 2}} \subset \mathscr{H}_{0}$. In order to extract the relevant terms as $\varepsilon \rightarrow 0$, we rescale the amplitudes $A_{0}, A_{1}$ and the parameter $\xi$, by defining $\eta=-\varepsilon \xi$ and setting $A_{0}(\xi)=\sqrt{\varepsilon} X_{0}(\eta), A_{1}(\xi)=\sqrt{\varepsilon} X_{1}(\eta), k(\sqrt{\varepsilon} x)=\sqrt{\varepsilon} l(x)$ for all $x \in \mathscr{H}_{0}$. We thus obtain the new system

$$
\begin{aligned}
& X_{0}^{\prime}=-\frac{\lambda_{0+}}{\varepsilon} X_{0}-\frac{1}{\lambda_{0+}-\lambda_{0-}} F_{0}(x+l(x)), \\
& X_{1}^{\prime}=-\frac{\lambda_{1+}}{\varepsilon} X_{1}-\frac{1}{\lambda_{1+}-\lambda_{1-}} F_{1}(x+l(x)),
\end{aligned}
$$

where $x=\left(X_{0}, X_{1}\right) \in \mathscr{B}_{R} \subset \mathscr{H}_{0}$ and ' denotes the derivative with respect to $\eta$. By construction, the function $l: \mathscr{H}_{0} \rightarrow \mathscr{H}$ has the same properties as $k$ in Corollary 3.4. In view of Eqs. (1.7), (3.1), (3.4) and Corollary 3.4, the formal limit $\varepsilon \rightarrow 0$ in Eq. (3.7) yields the simple equations

$$
\begin{aligned}
& X_{0}^{\prime}=\frac{1}{c-2 i} X_{0}\left(2-\left|X_{0}\right|^{2}-2\left|X_{1}\right|^{2}\right), \\
& X_{1}^{\prime}=\frac{1}{c+2 i} X_{1}\left(2 \alpha-\left|X_{1}\right|^{2}-2\left|X_{0}\right|^{2}\right) .
\end{aligned}
$$

We shall study these equations in the next subsection, and come back to the case $\varepsilon>0$ in Sect. 3.4.

3.3. The Limiting Case $\varepsilon=0$. We now study the reduced system (3.8) and show that it has front solutions. This system has two circles of fixed points corresponding to Eq. (2.4):

$$
\mathrm{F}_{2}^{0}=\left\{\left|X_{0}\right|=\sqrt{2}, X_{1}=0\right\}, \quad \mathrm{F}_{3}^{0}=\left\{X_{0}=0,\left|X_{1}\right|=\sqrt{2 \alpha}\right\} .
$$

Lemma 3.5. Every point of $\mathrm{F}_{3}^{0}$ is connected by an orbit to $\mathrm{F}_{2}^{0}$.

Proof. We begin by setting $X_{0}=\rho_{0} e^{i \psi_{0}}, X_{1}=\rho_{1} e^{i \psi_{1}}$, with $\rho_{0}, \rho_{1} \in \mathbf{R}_{+}$and $\psi_{0}, \psi_{1} \in \mathbf{R}$. Inserting in Eq. (3.8), we obtain the following equations for the amplitudes $\rho_{0}, \rho_{1}$ :

$$
(c+4 / c) \rho_{0}^{\prime}=\rho_{0}\left(2-\rho_{0}^{2}-2 \rho_{1}^{2}\right), \quad(c+4 / c) \rho_{1}^{\prime}=\rho_{1}\left(2 \alpha-\rho_{1}^{2}-2 \rho_{0}^{2}\right) .
$$

The equations for the phases $\psi_{0}, \psi_{1}$ can be explicitly integrated and yield the relations $\rho_{0}=C_{0} e^{c \psi_{0} / 2}, \rho_{1}=C_{1} e^{-c \psi_{1} / 2}$, where $C_{0}, C_{1}$ are positive constants determined by the initial conditions. We next eliminate the parameter $c$ from Eq. (3.9) by 
the transformation $r_{0}(\eta)=\rho_{0}((c+4 / c) \eta), r_{1}(\eta)=\rho_{1}((c+4 / c) \eta)$, leading to the system

$$
r_{0}^{\prime}=r_{0}\left(2-r_{0}^{2}-2 r_{1}^{2}\right), \quad r_{1}^{\prime}=r_{1}\left(2 \alpha-r_{1}^{2}-2 r_{0}^{2}\right) .
$$

Since $0<\alpha<1 / 2$ and $r_{0} \geqq 0, r_{1} \geqq 0$, it is straightforward to verify that Eq. (3.10) has exactly three fixed points: $F_{1}=(0,0)$ (a source), $F_{2}=(\sqrt{2}, 0)$ (a sink), and $\mathrm{F}_{3}=(0, \sqrt{2 \alpha})$ (a saddle).

We now prove the existence of a trajectory $\left(r_{0}, r_{1}\right)(\eta)$ of Eq. (3.10) leaving $F_{3}$ at $\eta=-\infty$ and reaching $F_{2}$ at $\eta=+\infty$. We will do this by showing that the (one-dimensional) unstable manifold $\mathscr{W}$ of $\mathrm{F}_{3}$ lies in the basin of attraction of $\mathrm{F}_{2}$. In order to do that, we consider the (closed) domain $D$ in $\mathbf{R}_{+} \times \mathbf{R}_{+}$bounded by the two curves

$$
E_{+}=\left\{\left(r_{0}, r_{1}\right) \mid r_{0}^{2}+2 r_{1}^{2}=2\right\}, \quad E_{-}=\left\{\left(r_{0}, r_{1}\right) \mid r_{1}^{2}+2 r_{0}^{2}=2 \alpha\right\},
$$

as shown in Fig. 5.

Elementary calculations show that, on both $E_{+}, E_{-}$, the vector field (3.10) points toward the interior of $D$, whereas it is parallel to the boundary on the two remaining segments of $\partial D$; this means that the interior $D$ of $D$ is invariant under the flow of Eq. (3.10). Moreover, it is easy to verify that $r_{0}^{\prime}>0$ and $r_{1}^{\prime}<0$ everywhere in $D$. As a consequence, since $D$ is compact, any trajectory in $D$ necessarily converges to some (fixed) point in $D$ as $\eta \rightarrow \infty$, and by elimination this fixed point must be $\mathrm{F}_{2}$. So, it remains to show that the unstable manifold $\mathscr{W}$ of $\mathrm{F}_{3}$ intersects $\stackrel{\circ}{D}$. Writing $\mathscr{W}=\left\{\left(r_{0}, f\left(r_{0}\right)\right) \mid r_{0}>0\right\}$ and $E_{-}=\left\{\left(r_{0}, \hat{f}\left(r_{0}\right)\right) \mid r_{0}>0\right\}$ near $r_{0}=0$, we easily find

$$
f\left(r_{0}\right)=\sqrt{2 \alpha}\left(1-\frac{1}{2} \frac{r_{0}^{2}}{1-\alpha}+\mathcal{O}\left(r_{0}^{4}\right)\right), \hat{f}\left(r_{0}\right)=\sqrt{2 \alpha}\left(1-\frac{1}{2} \frac{r_{0}^{2}}{\alpha}+\mathcal{O}\left(r_{0}^{4}\right)\right) .
$$

Since $1-\alpha>\alpha$, we have $f\left(r_{0}\right)>\hat{f}\left(r_{0}\right)$ if $r_{0}$ is sufficiently small, so that $\mathscr{W}$ lies in $D$ in a neighborhood of $F_{3}$.

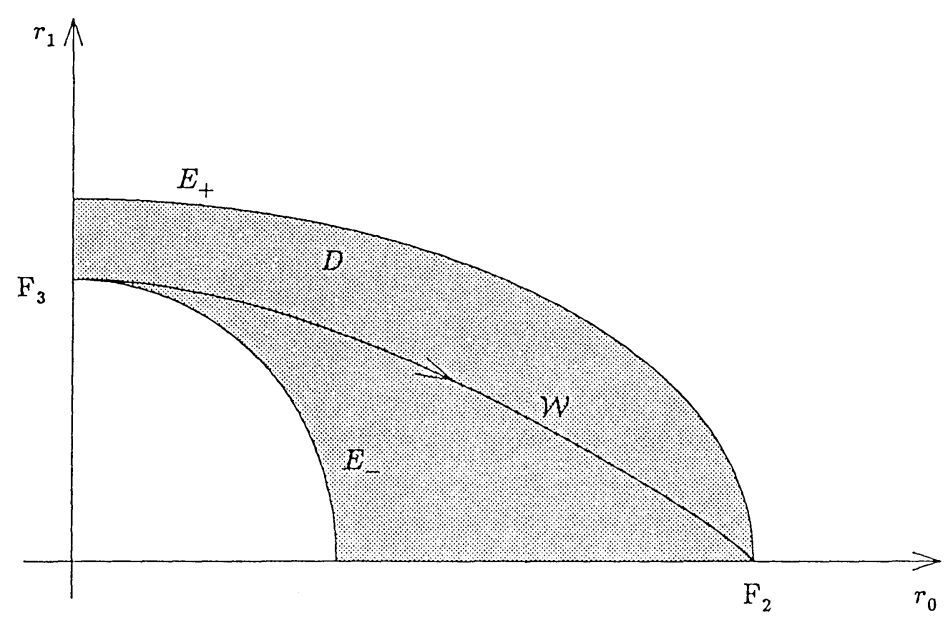

Fig. 5. The domain $D$ of $\mathbf{R}_{+} \times \mathbf{R}_{+}$bounded by the two ellipses $E_{+}, E_{-}$(shaded region) is attracted to $F_{2}$ by the flow of (3.10). In particular, the unstable manifold $\mathscr{W}$ of $F_{3}$ intersects $F_{2}$ 
Remark. The condition $\alpha<1 / 2$ is essential in this argument: if $1 / 2<\alpha<2$, both fixed points $\mathrm{F}_{2}, \mathrm{~F}_{3}$ are stable, so that no connection can occur between them. Note that the slope of the tangent to the curve $\left(q_{0}-q_{1}\right)^{2}=6 q_{1}^{2}-2$ at $q_{0}=-1, q_{1}=1$ is exactly $\frac{1}{2}$, see Fig. 2 .

3.4. The Case $\varepsilon>0$. We now come back to the full equations (3.7) and prove, by a perturbation argument, the existence of front solutions for sufficiently small $\varepsilon$, i.e., Theorem 1.1. Although this could be done by direct estimates in this simple finite-dimensional case, we shall use the general methods of Appendix A, as a preparation for the infinite-dimensional situation of Sect. 4.

The perturbation argument is a comparison of the flows $\Phi_{\eta}^{\varepsilon}, \Phi_{\eta}^{0}$ defined by the vector fields $\chi^{\varepsilon}, \chi^{0}$ of Eqs. (3.7), (3.8) respectively. By construction, the flow $\Phi_{n}^{\varepsilon}$ has two circles of fixed points:

$$
\begin{aligned}
& \left.\mathrm{F}_{2}^{\varepsilon}\right) \quad X_{0}=\frac{-\lambda_{0-}}{\lambda_{0+}-\lambda_{0}} \sqrt{2-\varepsilon} e^{i \varphi}, \quad X_{1}=0, \quad \varphi \in \mathbf{R}, \\
& \left.\mathrm{F}_{3}^{\varepsilon}\right) \quad X_{1}=\frac{-\lambda_{1-}}{\lambda_{1+}-\lambda_{1-}} \sqrt{2 \alpha-\alpha^{2} \varepsilon} e^{i \psi}, X_{0}=0, \quad \psi \in \mathbf{R} .
\end{aligned}
$$

Using Eqs. (3.1) and (3.3) it is easy to see that $\operatorname{dist}\left(\mathrm{F}_{2}^{\varepsilon}, \mathrm{F}_{2}^{0}\right)$ and $\operatorname{dist}\left(\mathrm{F}_{3}^{\varepsilon}, \mathrm{F}_{3}^{0}\right)$ are $\mathcal{O}(\varepsilon)$ as $\varepsilon \rightarrow 0$.

The main steps of the proof are:

i) The flows depend continuously on $\varepsilon$ at $\varepsilon=0$.

ii) The stable and unstable manifolds of $\mathrm{F}_{2}^{\varepsilon}, \mathrm{F}_{3}^{\varepsilon}$ are continuous in $\varepsilon$ at $\varepsilon=0$.

We begin by comparing the flows.

Proposition 3.6. There exists a $K_{3}>0$ such that

$$
\left\|\Phi_{\eta}^{\varepsilon}(x)-\Phi_{\eta}^{0}(y)\right\| \leqq \exp \left(\frac{K_{3} \eta}{c+2}\right)\left(\|x-y\|+K_{3}\left(\varepsilon / \varepsilon_{c}\right)\right),
$$

for all $x, y \in \mathscr{B}_{R} \subset \mathscr{H}_{0}$ and all $\eta \in \mathbf{R}_{+}$.

Proof. We first note that $\chi^{\varepsilon}, \chi^{0}$ are close to each other in the Lipschitz norm:

Lemma 3.7. Let $\Delta \chi(x)=\chi^{\varepsilon}(x)-\chi^{0}(x), x \in \mathscr{H}_{0}$. Then, there exists a $K_{4}>0$ such that $\operatorname{Lip}(\Delta \chi) \leqq\left(\varepsilon / \varepsilon_{c}\right) K_{4} /(c+2)$ in $\mathscr{B}_{2 R} \subset \mathscr{H}_{0}$.

The proof is a calculation which can be found in Appendix B.

We next write Eq. (3.7) in the form $x^{\prime}=\chi^{0}(x)+\Delta \chi(x)$, regarding $\Delta \chi$ as an additional non-linear term. From this point of view, the systems (3.7), (3.8) have the same linear part, with spectrum contained in the half-plane $\operatorname{Re}(z) \leqq 2 c /\left(c^{2}+4\right)$. The non-linear part of $\chi^{0}$ has (in $\left.\mathscr{B}_{2 R}\right)$ a Lipschitz constant bounded by $C /(c+2)$, for some $C>0$, and by Lemma 3.7 the same is true for $\chi^{\varepsilon}=\chi^{0}+\Delta \chi$, with $C$ replaced by $C+K_{4}\left(\varepsilon / \varepsilon_{c}\right)$. So, setting $\mathscr{E}^{c s}=\mathscr{H}_{0}, \mathscr{E}^{u}=\{0\}, \lambda^{c s}=2 c /\left(c^{2}+4\right)$, $D=1$, and $\ell^{c s}(2 R)=\left(C+K_{4}\right) /(c+2)$, we can apply Theorem A.1 to both systems simultaneously, the condition (A.4) being fulfilled with $\beta=K_{3} /(c+2)$ for some sufficiently large $K_{3}>0$. It follows that $\Phi^{\varepsilon}, \Phi^{0} \in \mathscr{K}_{\beta}$, and in particular we have $\left\|\Phi_{\eta}^{\varepsilon}(x)-\Phi_{\eta}^{\varepsilon}(y)\right\| \leqq e^{\beta \eta}\|x-y\|$ for all $x, y \in \mathscr{B}_{R}$ and all $\eta \in \mathbf{R}_{+}$. Now, we apply 
Theorem A.2 to compare $\Phi^{\varepsilon}$ with $\Phi^{0}$, the condition (A.5) being fulfilled with $A_{1}^{c s}=A_{2}^{c s}, f_{1}^{c s}-f_{2}^{c s}=\Delta \chi, \delta=0$ and $\varepsilon \rightarrow \varepsilon / \varepsilon_{c}$. By Eq. (A.6), we thus have $\left\|\Phi_{\eta}^{\varepsilon}(y)-\Phi_{\eta}^{0}(y)\right\| \leqq(1-2 \sigma)^{-1} R\left(\varepsilon / \varepsilon_{c}\right) e^{\beta \eta}$ for all $y \in \mathscr{B}_{R}$ and all $\eta \in \mathbf{R}_{+}$. Combining these results, we obtain Eq. (3.11).

We now study the stable and unstable manifolds of $\mathrm{F}_{2}^{\varepsilon}, \mathrm{F}_{3}^{\varepsilon}$.

Lemma 3.8. There is a $K_{5}>0$ such that, for $\varepsilon / \varepsilon_{c} \leqq K_{5} c /(c+2)$, the annulus of radius $\rho_{2}=K_{5} c /(c+2)$ around the circle $\mathrm{F}_{2}^{\varepsilon}$ is attracted to $\mathrm{F}_{2}^{\varepsilon}$ by the flow $\Phi_{\eta}^{\varepsilon}$.

Proof. We first study the geometry in the case $\varepsilon=0$. Let $x^{0}=(\sqrt{2}, 0) \in \mathrm{F}_{2}^{0}$ and set $x=x^{0}+z$, with $z=\left(Z_{0}, Z_{1}\right) \in \mathscr{B}_{2 \rho} \subset \mathscr{H}_{0}$ for sufficiently small $\rho>0$. Inserting in Eq. (3.8), we obtain

$$
Z_{0}^{\prime}=\frac{-2}{c-2 i}\left(Z_{0}+Z_{0}^{*}\right)+f_{0}(z), \quad Z_{1}^{\prime}=\frac{2 \alpha-4}{c+2 i} Z_{1}+f_{1}(z)
$$

where $f: \mathscr{H}_{0} \rightarrow \mathscr{H}_{0}$ is $\mathscr{C}^{1,1}$ and $\operatorname{Lip}(f) \leqq C \rho /(c+2)$ in $\mathscr{B}_{2 \rho}$ for some $C>0$. As is easily verified, the linear part of Eq. (3.12) (regarded as an operator in $\mathbf{R}^{4}$ ) has one zero and three stable eigenvalues. The gap between the stable and the central part of the spectrum is equal to $2 c /\left(c^{2}+4\right)$, and if $V_{s}, V_{c}$ denote the corresponding eigenspaces, then $x^{0}+V_{c}$ is just the tangent to the circle $\mathrm{F}_{2}^{0}$ at $x^{0}$, and $V_{s}$ is the normal hyperplane. This situation prevails for small $\varepsilon$. To see this, denote by $x^{\varepsilon}$ the unique point of $\mathrm{F}_{2}^{\varepsilon}$ for which $X_{0} \in \mathbf{R}_{+}$. Setting now $x=x^{\varepsilon}+z$ and inserting in Eq. (3.7), we obtain Eq. (3.12), with $f^{\varepsilon}(z)=f(z)+\delta \chi(z)$ replacing $f(z)$, where $\delta \chi(z)=\chi^{\varepsilon}\left(x^{\varepsilon}+z\right)-\chi^{0}\left(x^{0}+z\right)$. In Appendix B, we prove:

Lemma 3.9. There exists a $K_{6}>0$ such that $\operatorname{Lip}(\delta \chi) \leqq\left(\varepsilon / \varepsilon_{c}\right) K_{6} /(c+2)$ in $\mathscr{B}_{R} \subset \mathscr{H}_{0}$.

Using this bound, we can compare the two systems (i.e., Eq. (3.12) with $f$ or $f^{\varepsilon}$ in the right-hand side) in the ball $\mathscr{B}_{\rho} \subset \mathscr{H}_{0}$. Setting $\mathscr{E} c s=V_{s}, \mathscr{E}^{u}=V_{c}, \lambda^{c s}=$ $-2 c /\left(c^{2}+4\right), \lambda^{u}=0, D=1$ and $\ell^{c s}(2 \rho), \ell^{u}(2 \rho)=\left(C \rho+K_{6} \varepsilon / \varepsilon_{c}\right) /(c+2)$, the condition (A.3) can be fulfilled if $\varepsilon / \varepsilon_{c} \leqq \rho$ and $\rho \leqq \rho_{2}=K_{5} c /(c+2)$ for some (sufficiently small) $K_{5}>0$. Thus, by Theorem A.1, there exist $h^{0}, h^{\varepsilon}: \mathscr{B}_{\rho_{2}} \subset V_{s} \rightarrow V_{c}$ whose graphs $\mathscr{V}^{0}, \mathscr{V}^{\varepsilon}$ are (except for a translation) the local stable manifolds of $x^{0}, x^{\varepsilon}$ for the flows $\Phi_{\eta}^{0}, \Phi_{\eta}^{\varepsilon}$ respectively. Moreover, applying Theorem A.2 with $A_{1}=A_{2}, f_{1}-f_{2}=\delta \chi, \delta=0$ and $\varepsilon \rightarrow \varepsilon / \varepsilon_{c}$, we easily see that $\left\|h^{\varepsilon}-h^{0}\right\|_{\infty}=\mathcal{O}\left(\varepsilon / \varepsilon_{c}\right)$ in $\mathscr{B}_{\rho_{2}} \subset V_{s}$.

Note that, in view of the phase covariance of the system, we can obtain from $\mathscr{V}^{\varepsilon}$ the corresponding stable manifold of any point of $F_{2}^{\varepsilon}$ by simply applying the transformation $X_{0} \rightarrow X_{0} e^{i \varphi}, \varphi \in \mathbf{R}$. The union over $\varphi \in[0,2 \pi]$ of these manifolds is the annulus of radius $\rho_{2}$ around the circle $F_{2}^{\varepsilon}$. This completes the proof of Lemma 3.8.

These considerations about the behavior of $\Phi_{\eta}^{0}, \Phi_{\eta}^{\varepsilon}$ near $\mathrm{F}_{2}^{0}, \mathrm{~F}_{2}^{\varepsilon}$ can be repeated in an analogous manner for $\mathrm{F}_{3}^{0}, \mathrm{~F}_{3}^{\varepsilon}$. Choosing $y^{0}=(0, \sqrt{2 \alpha}) \in \mathrm{F}_{3}^{0}$ and setting $x=y^{0}+z$, we obtain instead of Eq. (3.12),

$$
Z_{0}^{\prime}=\frac{2-4 \alpha}{c-2 i} Z_{0}+g_{0}(z), \quad Z_{1}^{\prime}=\frac{-2 \alpha}{c+2 i}\left(Z_{1}+Z_{1}^{*}\right)+g_{1}(z),
$$

where again $\operatorname{Lip}(g) \leqq C \rho /(c+2)$ in $\mathscr{B}_{2 \rho} \subset \mathscr{H}_{0}$. Recalling that $\alpha<1 / 2$, one verifies that the linear part has one stable, one zero and two unstable eigenvalues. The gap 
between the center-stable and the unstable part of the spectrum is equal to $(2-4 \alpha) c /\left(c^{2}+4\right)$, and if $W_{c s}, W_{u}$ denote the corresponding eigenspaces, then $W_{u}$ is just the complex line $\left\{Z_{1}=0\right\}$. In the same way, choosing $y^{\varepsilon} \in \mathrm{F}_{3}^{\varepsilon}$ as above and setting $x=y^{\varepsilon}+z$, we obtain Eq. (3.13) with $g$ replaced by some $g^{\varepsilon}$ such that $\operatorname{Lip}\left(g^{\varepsilon}-g\right) \leqq K_{6}\left(\varepsilon / \varepsilon_{c}\right) /(c+2)$. So, reversing the sign of the "time" $\eta$ and setting $\mathscr{E}^{c s}=W_{u}, \mathscr{E}^{u}=W_{c s}, \quad \lambda^{c s}=-(2-4 \alpha) c /\left(c^{2}+4\right), \quad \lambda^{u}=0, \quad D=1, \quad$ and $\ell^{c s}(2 \rho)$, $\ell^{u}(2 \rho)=\left(C \rho+K_{6} \varepsilon / \varepsilon_{c}\right) /(c+2)$, the condition (A.3) can be fulfilled if $\varepsilon / \varepsilon_{c} \leqq \rho$ and $\rho \leqq \rho_{3}=K_{5}(1-2 \alpha) c /(c+2)$. Thus, there exist $k^{0}, k^{\varepsilon}: \mathscr{B}_{\rho_{3}} \subset W_{u} \rightarrow W_{c s}$ whose graphs $\mathscr{W}^{0}, \mathscr{W}^{\varepsilon}$ are (except for a translation) the local unstable manifolds of $y^{0}, y^{\varepsilon}$; moreover $\left\|k^{\varepsilon}-k^{0}\right\|_{\infty}=\mathcal{O}\left(\varepsilon / \varepsilon_{c}\right)$ in $\mathscr{B}_{\rho_{3}} \subset W_{u}$.

Having gained control near the circle of fixed points, we can conclude the proof of Theorem 1.1 by following the flow in the space in between, using Proposition 3.6. Assuming that $\varepsilon / \varepsilon_{c} \leqq \rho_{3}$, we choose two points in the unstable manifolds of $\mathrm{F}_{3}^{\varepsilon}$ and $\mathrm{F}_{3}^{0}$, defined by

$$
P^{\varepsilon}=y^{\varepsilon}+\left(\rho_{3}, k^{\varepsilon}\left(\rho_{3}\right)\right) \in y^{\varepsilon}+\mathscr{W}^{\varepsilon}, \quad P^{0}=y^{0}+\left(\rho_{3}, k^{0}\left(\rho_{3}\right)\right) \in y^{0}+\mathscr{W}^{0} .
$$

By construction, $\operatorname{dist}\left(P^{\varepsilon}, P^{0}\right) \leqq\left\|y^{\varepsilon}-y^{0}\right\|+\left|k^{\varepsilon}\left(\rho_{3}\right)-k^{0}\left(\rho_{3}\right)\right|=\mathcal{O}\left(\varepsilon / \varepsilon_{c}\right) \quad$ as $\varepsilon \rightarrow 0$. On the other hand, since $P^{0}$ lies in the unstable manifold of $\mathrm{F}_{3}^{0}$, we have seen in Sect. 3.3 that $\Phi_{\eta}^{0}\left(P^{0}\right)$ converges to $\mathrm{F}_{2}^{0}$ as $\eta \rightarrow \infty$. Thus, there exists an $\eta>0$ such that $\operatorname{dist}\left(\Phi_{\eta}^{0}\left(P^{0}\right), \mathrm{F}_{2}^{0}\right) \leqq \rho_{2} / 3$, and it follows from Eq. (3.9) that $\eta=(c+4 / c) T$, where $T=T\left(\rho_{2}, \rho_{3}\right)$ does not depend explicitly on $c$. Finally, we know from Eq. (3.11) that

$$
\operatorname{dist}\left(\Phi_{\eta}^{\varepsilon}\left(P^{\varepsilon}\right), \Phi_{\eta}^{0}\left(P^{0}\right)\right) \leqq \exp \left(K_{3} \frac{c+4 / c}{c+2} T\right)\left(\operatorname{dist}\left(P^{\varepsilon}, P^{0}\right)+K_{3}\left(\varepsilon / \varepsilon_{c}\right)\right) .
$$

Now, let us choose $\varepsilon$ so small that $\operatorname{dist}\left(\Phi_{\eta}^{\varepsilon}\left(P^{\varepsilon}\right), \Phi_{\eta}^{0}\left(P^{0}\right)\right)$ and $\operatorname{dist}\left(\mathrm{F}_{2}^{\varepsilon}, \mathrm{F}_{2}^{0}\right)$ are smaller than $\rho_{2} / 3$. Then, by the triangle inequality

$$
\begin{aligned}
\operatorname{dist}\left(\Phi_{\eta}^{\varepsilon}\left(P^{\varepsilon}\right), \mathrm{F}_{2}^{\varepsilon}\right) & \leqq \operatorname{dist}\left(\Phi_{\eta}^{\varepsilon}\left(P^{\varepsilon}\right), \Phi_{\eta}^{0}\left(P^{0}\right)\right)+\operatorname{dist}\left(\Phi_{\eta}^{0}\left(P^{0}\right), \mathrm{F}_{2}^{0}\right)+\operatorname{dist}\left(\mathrm{F}_{2}^{0}, \mathrm{~F}_{2}^{\varepsilon}\right) \\
& \leqq \rho_{2} .
\end{aligned}
$$

In view of Lemma 3.8 , this means that $\Phi_{\eta}^{\varepsilon}\left(P^{\varepsilon}\right) \rightarrow \mathrm{F}_{2}^{\varepsilon}$ as $\eta \rightarrow \infty$, while $\Phi_{\eta}^{\varepsilon}\left(P^{\varepsilon}\right) \rightarrow y^{\varepsilon} \in \mathrm{F}_{3}^{\varepsilon}$ as $\eta \rightarrow-\infty$ since $P^{\varepsilon} \in y^{\varepsilon}+\mathscr{W}^{\varepsilon}$. Thus, we have shown the existence of a solution of Eq. (3.7) connecting $y^{\varepsilon} \in \mathrm{F}_{3}^{\varepsilon}$ to some point of $\mathrm{F}_{2}^{\varepsilon}$. The various assumptions on $\varepsilon$ can be summarized by the single condition $\varepsilon \leqq \varepsilon_{1}(c)$, where

$$
\varepsilon_{1}(c)=K_{7}\left(\frac{c}{c+2}\right)^{2} \exp \left(-K_{3} \frac{c+2}{c} T\left(\rho_{2}, \rho_{3}\right)\right),
$$

for some $K_{7}>0$. Since $\rho_{2}, \rho_{3}$ have positive limits as $c \rightarrow \infty$, so does $\varepsilon_{1}(c)$. This concludes the proof of Theorem 1.1.

\section{The Stable-Unstable Case}

We now study the more interesting case where one of the stationary solutions is (Eckhaus) stable and the other unstable, i.e., we choose two wave-numbers $q_{0}, q_{1}$ such that $q_{0}^{2}<1 / 3,1 / \sqrt{3}<q_{1}<1$. We follow the procedure announced in Sect. 2.2. 
4.1. Reduction to the Center-Unstable Manifold. We first investigate the behavior of the spectrum (2.1) as $c \rightarrow \infty$. If $n \in \mathbf{Z}$ is fixed and if $c \geqq 8\left(1+\left|q_{n}\right|\right)$, we find by straightforward calculations

$$
\lambda_{n+}=\frac{1}{c}\left(q_{n}^{2}-1\right)+\frac{1}{c^{2}} \mathscr{R}\left(c, q_{n}\right),
$$

where $\left|\mathscr{R}\left(c, q_{n}\right)\right| \leqq 5\left(1+\left|q_{n}\right|\right)^{3}$. On the other hand, if $c \geqq 2$, it is easy to see that $\operatorname{Re} \lambda_{n+} \geqq-\hat{\lambda}$ for all $n \in \mathbf{Z}$, where

$$
\hat{\lambda}=\frac{1}{2}\left(c-\sqrt{c^{2}-4}\right)=\frac{1}{c}+\mathcal{O}\left(\frac{1}{c^{3}}\right) .
$$

Since $\lambda_{n+}+\lambda_{n-}=-\left(c+2 i q_{n}\right)$, it follows that $\operatorname{Re} \lambda_{n-} \leqq-c+\hat{\lambda}$, so that the gap between the two branches of the spectrum is greater than $c-2 \hat{\lambda}=\sqrt{c^{2}-4}$. As a consequence, we have for all $n \in \mathbf{Z}$,

$$
\frac{1}{\left|\lambda_{n+}-\lambda_{n-}\right|} \leqq \frac{1}{\sqrt{c^{2}-4}}=\mathcal{O}\left(\frac{1}{c}\right) \text {. }
$$

Finally, if $n$ is fixed and $c \geqq 8\left(1+\left|q_{n}\right|\right)$, we find

$$
\left|\frac{1}{\lambda_{n+}-\lambda_{n-}}-\frac{1}{c}\right| \leqq \frac{4}{c^{2}}\left(1+\left|q_{n}\right|\right) .
$$

We now follow exactly the same procedure as in Sect. 3.2: reversing the sign of the time $\xi$ in (2.3), we apply Theorem A.1 to show the existence of a center-stable manifold corresponding to the branch $\left\{-\lambda_{n+}\right\}_{n \in Z}$ of the spectrum. Using the same notation, we take $\mathscr{E}^{c s}=\mathscr{E} u=\mathscr{H}, \lambda^{c s}=\hat{\lambda}, \lambda^{u}=c-\hat{\lambda}, D=1$, and $\beta=c / 3$. Moreover, if $c$ is sufficiently large, we see from Eqs. (2.5), (4.3) that $\ell^{c s}(r)=\ell^{u}(r) \leqq C r^{2} / c$ for some $C>0$. So, defining $r_{1}=K_{1} c$ for some (sufficiently small) $K_{1}>0$, we have $\sigma(r, \beta) \leqq \frac{1}{2}\left(r / r_{1}\right)^{2}$ in Eq. (A.2), and Lemma 3.2 still holds for $r<r_{1}$. This shows the existence of the local center-unstable manifold $\Gamma_{r}=\left\{(A, B) \in \mathscr{H}^{2} \mid B=h(A), A \in \mathscr{B}_{r} \subset \mathscr{H}\right\}$ for the system (2.3). Now, as is easily seen from Eqs. (4.1), (4.3), the fixed points (2.4) have a finite limit as $c \rightarrow \infty$; so, we can find an $R>0$ such that $\mathrm{F}_{2}, \mathrm{~F}_{3} \in \mathscr{B}_{R} \subset \mathscr{H}^{2}$ for all sufficiently large $c$. Defining thus $c_{0}=R / K_{1}$ and noting that $\frac{1}{2}\left(R / r_{1}\right)^{2}=\frac{1}{2}\left(c_{0} / c\right)^{2}$, we obtain:

Proposition 4.1. There exists a $c_{0}>0$ such that, for all $c \geqq c_{0}$, the system (2.3) defines a semiflow (for $\xi \leqq 0$ ) on the local center-unstable manifold $\Gamma_{R}$, which contains the fixed points (2.4). The projection onto $\mathscr{H}$ of this semiflow verifies the differential equation (3.5), with $h: \mathscr{H} \rightarrow \mathscr{H}$ a phase covariant $C^{1,1}$ function verifying $h(0)=0, D h(0)=0, \operatorname{Lip}(h) \leqq \frac{1}{2}\left(c_{0} / c\right)^{2}$.

This proposition reduces the proof of Theorem 1.2 to the study of the system (3.5) in the ball $\mathscr{B}_{R} \subset \mathscr{H}$. In order to extract the leading terms as $c \rightarrow \infty$, we rescale the time $\xi$ by setting $\xi=-c \eta$. We thus obtain

$$
A_{n}^{\prime}=\alpha_{n}(c) A_{n}-v_{n}(c) F_{n}(A+h(A)), \quad A \in \mathscr{B}_{R} \subset \mathscr{H},
$$

where $\alpha_{n}(c)=-c \lambda_{n+}, v_{n}(c)=c /\left(\lambda_{n+}-\lambda_{n-}\right)$, and 'denotes the derivative with respect to $\eta$. In view of Eqs. (4.1), (4.4), the formal limit $c \rightarrow \infty$ in Eq. (4.5) yields the simpler equations

$$
A_{n}^{\prime}=\alpha_{n} A_{n}-F_{n}(A), \quad A \in \mathscr{B}_{R} \subset \mathscr{H},
$$

where $\alpha_{n}=1-q_{n}^{2}$. 
4.2. The Limiting Case $c=\infty$. We now study the reduced equations (4.6) and show that they have front solutions. Of course, this system is still infinitedimensional, so that we cannot hope to show the existence of front solutions just by a simple argument as in Sect. 3.3. For convenience, we suppose from now on that $-1 / \sqrt{3}<q_{0} \leqq 0$ and that $q_{1}=\sqrt{1-\varepsilon^{2}}$ for some $\varepsilon \leqq \varepsilon_{0}=1 / 10$ (Fig. 2, black region). Then, recalling that $q_{n}=q_{0}+n\left(q_{1}-q_{0}\right)$ and $\alpha_{n}=1-q_{n}^{2}$, we see that $\alpha_{0}, \alpha_{1}$ (and perhaps $\alpha_{-1}$ ) are positive, whereas $\alpha_{n}<0$ for all $|n|>1$. This means that most of the variables $A_{n}$ are exponentially damped by Eq. (4.6), so that only a few modes $\left(A_{-1}, A_{0}, A_{1}\right)$ will be relevant in our analysis.

We first consider the behavior of the system in a neighborhood of the two circles of fixed points $F_{2}, F_{3}$ corresponding to Eq. (2.4):

$\left.\mathrm{F}_{2}\right) \quad\left|A_{0}\right|=\sqrt{\alpha_{0}}, A_{n}=0$ for all $n \neq 0$,

$\left.\mathrm{F}_{3}\right) \quad\left|A_{1}\right|=\sqrt{\alpha_{1}}, A_{n}=0$ for all $n \neq 1$.

The following results will be proven in Sect. 4.3:

i) The circle $\mathrm{F}_{2}$ has an annular neighborhood $\mathscr{A}$ which is attracted to $\mathrm{F}_{2}$ by Eq. (4.6) and whose size does not depend on $\varepsilon$.

ii) Any point $P \in \mathrm{F}_{3}$ has a local unstable manifold $\mathscr{W}_{P}$ of (complex) dimension 1, which is nearly parallel to the 0 -direction (i.e., the direction defined by $A_{0}=1$, $A_{n}=0$ for all $n \neq 0$ ), and whose size does not depend on $\varepsilon$.

To prove that front solutions exist for $c=\infty$ we now show that the continuation of the local unstable manifold $\mathscr{W}_{P}$ under the semiflow defined by Eq. (4.6) intersects the attractive annular neighborhood $\mathscr{A}$, if $\varepsilon$ is sufficiently small. This has to be done by direct estimates; for the sake of clarity, we just explain here the main steps of the calculation, and defer the proofs to Appendix C.

First of all, we write any $A \in \mathscr{H}$ as $A_{\|}+A_{\perp}$, where $A_{\|}=\left(A_{-1}, A_{0}, A_{1}\right)$ and $A_{\perp}=\left(A_{n}\right)_{|n|>1}$; the corresponding decomposition of $\mathscr{H}$ will be denoted by $\mathscr{H}_{||} \oplus \mathscr{H}_{\perp}$. We also define the domain $D_{\varepsilon}=\left\{A_{\|}|| A_{0}|\leqq 1,| A_{1}|\leqq 2 \varepsilon,| A_{-1} \mid \leqq 2 \varepsilon\right\}$ $\subset \mathscr{H}_{\|}$, and note that the fixed points $\mathrm{F}_{2}, \mathrm{~F}_{3}$ lie in $D_{\varepsilon}$, see Fig. $\overline{6}$.

Now, if $A(\eta)$ is a solution of Eq. (4.6) and if $\varepsilon$ is sufficiently small, we have the following results:

Lemma 4.2. There exists a $K_{2}>0$ such that, if $A_{\|} \in D_{\varepsilon}$ and $\left\|A_{\perp}\right\|=K_{2} \varepsilon^{2}$, then $\frac{d}{d \eta}\left\|A_{\perp}\right\|<0$.

In other words, as long as $A_{\|}$stays in $D_{\varepsilon}$, the other components $A_{\perp}$ of $A$ remain bounded by $K_{2} \varepsilon^{2}$ if they were initially. Since we are interested in a trajectory starting from $\mathrm{F}_{3}$ (where $A_{\perp}=0$ ), we can henceforth assume that $\left\|A_{\perp}\right\| \leqq K_{2} \varepsilon^{2}$, as long as $A_{\|} \in D_{\varepsilon}$.

Lemma 4.3. If $\left\|A_{\perp}\right\| \leqq K_{2} \varepsilon^{2}, A_{\|} \in D_{\varepsilon}$ and $\left|A_{1}\right|=2 \varepsilon$, then $\frac{d}{d \eta}\left|A_{1}\right|<0$.

A similar result holds for $A_{-1}$ replacing $A_{1}$. Together with Lemma 4.2, this means that a trajectory of the system cannot leave the region $\hat{D}_{\varepsilon}=$ $\left\{A_{\|} \in D_{\varepsilon},\left\|A_{\perp}\right\| \leqq K_{2} \varepsilon^{2}\right\}$ unless $\left|A_{0}\right|>1$. In particular, we can assume in our case that $A \in \hat{D}_{\varepsilon}$, as long as $\left|A_{0}\right| \leqq 1$. 


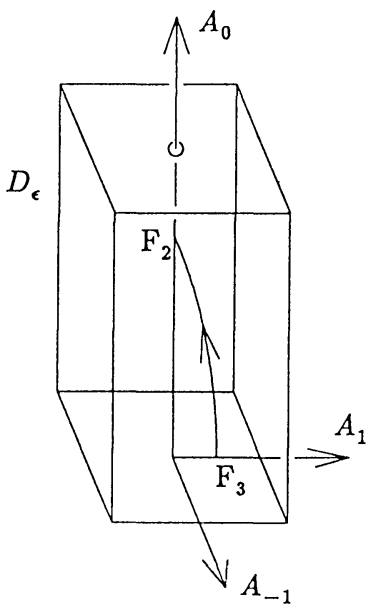

Fig. 6. The parallelepiped $D_{\varepsilon}$ contains the fixed points $F_{2}, F_{3}$. The unstable manifold of $\mathrm{F}_{3}$ remains in this domain and goes along the $A_{0}$-axis until it intersects the basin of attraction of $\mathrm{F}_{2}$ Lemma 4.4. If $A \in \hat{D_{\varepsilon}}$ and $\varepsilon^{3} \leqq\left|A_{0}\right| \leqq \sqrt{\alpha_{0}}(1-\varepsilon)$, then $\frac{d}{d \eta}\left|A_{0}\right|$ is positive and
bounded away from zero.

Now, the main result of this subsection is:

Proposition 4.5. There is an $\varepsilon_{1} \leqq \varepsilon_{0}$ such that, for all $\varepsilon \leqq \varepsilon_{1}$, every point of $\mathrm{F}_{3}$ is connected to $\mathrm{F}_{2}$ by an orbit of the system (4.6).

Proof. If $\mathscr{W}_{P}$ is the local unstable manifold of $P \in \mathrm{F}_{3}$, we can choose $A \in \mathscr{W}_{P} \cap \hat{D}_{\varepsilon}$ such that $\left|A_{0}\right| \geqq \varepsilon^{3}$. This is always possible if $\varepsilon$ is sufficiently small, because $\mathscr{W}_{P}$ is nearly tangent to the 0 -direction and its size does not depend on $\varepsilon$. So, denoting by $A(\eta)(\eta \geqq 0)$ the evolution of $A$ under Eq. (4.6), we know from the preceding lemmas that $A(\eta)$ remains in $\hat{D}_{\varepsilon}$ and is driven along the 0 -direction with non-vanishing velocity until $\left|A_{0}(\eta)\right|=\sqrt{\alpha}_{0}(1-\varepsilon)$. But, as is easily verified, this last point lies in the annular neighborhood $\mathscr{A}$ of the circle $\mathrm{F}_{2}$, if $\varepsilon$ is sufficiently small.

Remarks.

1) If $A(\eta)$ is a solution of Eq. (4.6) and if

$$
u(x, t)=\sum_{n \in \mathbf{Z}} A_{n}(t) e^{i q_{n} x},
$$

then it is easy to see that $u(x, t)$ verifies the GL-equation (1.1). So, Proposition 4.5 shows the existence of solutions of Eq. (1.1) satisfying

$$
\lim _{t \rightarrow-\infty} u(x, t)=u_{q_{1}}(x), \quad \lim _{t \rightarrow+\infty} u(x, t)=u_{q_{0}}(x),
$$

uniformly in $x$ (unlike the front solutions).

2) Let $n$ be the (real) dimension of the unstable manifold of any point of $F_{3}$, and let $m$ be the (real) codimension of the stable manifold of the circle $\mathrm{F}_{2}$. For $q_{0}, q_{1}$ in the range of Theorem 1.2, we have seen that $n=2$ and $m=0$, so that the intersection of the two manifolds is generic in the sense that $n>m$. For other values of $q_{0}, q_{1}$, it is not difficult to show that this genericity condition is fulfilled if and only if $\left(q_{0}-q_{1}\right)^{2}<6 q_{1}^{2}-2$. This is the shaded region in Fig. 2. 
4.3. The Full Case $c<\infty$. We now come back to the full equations (4.5) and show, by the same perturbation argument as in Sect. 3.4, the existence of front solutions for sufficiently large $c \geqq c_{0}$. To simplify the forthcoming expressions, we rewrite Eqs. (4.5), (4.6) in the form

$$
\begin{aligned}
& A^{\prime}=\Lambda^{c} A-F^{c}(A), \quad A \in \mathscr{B}_{R} \subset \mathscr{H}, \\
& A^{\prime}=\Lambda A-F(A), \quad A \in \mathscr{B}_{R} \subset \mathscr{H},
\end{aligned}
$$

where $\Lambda^{c}, \Lambda$ are the linear operators in $\mathscr{H}$ defined by $\left(\Lambda^{c} A\right)_{n}=\alpha_{n}(c) A_{n}$, $(\Lambda A)_{n}=\alpha_{n} A_{n}$, and $F^{c}: \mathscr{H} \rightarrow \mathscr{H}$ is the $\mathscr{C}^{1,1}$ function defined by $\left(F^{c}(A)\right)_{n}$ $=v_{n}(c) F_{n}(A+h(A))$.

We begin by comparing the semiflows $\Phi_{\eta}^{c}, \Phi_{\eta}$ of Eq. $\left(4.5^{\prime}\right),\left(4.6^{\prime}\right)$ respectively.

Proposition 4.6. There exists a $K_{3}>0$ such that,

$$
\left\|\Phi_{\eta}^{c}(A)-\Phi_{\eta}(B)\right\| \leqq \exp \left(K_{3} \eta\right)\left(\|A-B\|+\frac{K_{3}}{c^{1 / 4}}\right),
$$

for all $A, B \in \mathscr{B}_{R} \subset \mathscr{H}$ and all $\eta \geqq c^{-1 / 4}$.

Proof. Since (by Eqs. (4.1), (4.2)) $\alpha_{n} \leqq 1$ and $\operatorname{Re} \alpha_{n}(c) \leqq c \hat{\lambda}$ for all $n \in \mathbf{Z}$, there exists a $\lambda>1$ such that $\left\|e^{\Lambda^{c} t}\right\|,\left\|e^{\Lambda t}\right\| \leqq e^{\lambda t}$ for all $t \in \mathbf{R}_{+}$and all $c \geqq c_{0}$. On the other hand, using Eqs. (2.5), (4.3) and Proposition 4.1, it is easy to see that $\operatorname{Lip}\left(F^{c}\right)$, $\operatorname{Lip}(F) \leqq K_{4}$ in $\mathscr{B}_{2 R} \subset \mathscr{H}$, for some $K_{4}>0$. So, setting $\mathscr{E} c s=\mathscr{H}, \mathscr{E} u=\{0\}$, $\lambda^{c s}=\lambda, D=1$, and $\ell^{c s}(2 R)=K_{4}$, we can apply Theorem A.1 to both systems $\left(4.5^{\prime}\right)$, $\left(4.6^{\prime}\right)$, the condition (A.3) being fulfilled if $\beta>\lambda+10 K_{4}$. It follows that $\Phi^{c}, \Phi \in \mathscr{K}_{\beta}$ for all $c \geqq c_{0}$.

Now, we want to use Theorem A.2 to compare the semiflows $\Phi^{c}, \Phi$ in $\mathscr{B}_{R} \subset \mathscr{H}$. Before doing this, let us remark that, although (by Eq. (4.1)) $\alpha_{n}(c) \equiv-c \lambda_{n+}$ converges to $\alpha_{n}=1-q_{n}^{2}$ for all $n$ as $c \rightarrow \infty$, the convergence is not uniform in $n$ : in fact, $\left|\alpha_{n}(c)\right|$ grows like $\sqrt{n}$ as $n \rightarrow \infty$ and $\left|\alpha_{n}\right|$ like $n^{2}$, so that $\left\|\Lambda^{c}-\Lambda\right\|=\infty$ for all $c$. On the other hand, the conditions (A.5) do not involve the operators themselves, but the associated semigroups $e^{\Lambda^{c} t}, e^{\Lambda t}$, in which the large $n$ components are exponentially small if $t>0$. So, we can hope that $e^{\Lambda^{c} t}, e^{\Lambda t}$ are close to each other if $c$ is sufficiently large and $t$ strictly positive. Indeed, we find:

\section{Lemma 4.7.}

$$
\sup _{t \geqq c^{-1 / 4}}\left(e^{-\lambda t}\left\|e^{\Lambda^{c} t}-e^{\Lambda t}\right\|\right)=\mathcal{O}\left(\frac{1}{c^{1 / 4}}\right), \text { as } c \rightarrow \infty .
$$

(See Appendix $C$ for the proofs of the lemmas in this section.) The same phenomenon occurs when comparing the non-linear terms $F^{c}, F$, for the convergence of the factor $v_{n}(c) \equiv c /\left(\lambda_{n+}-\lambda_{n-}\right)$ to 1 as $c \rightarrow \infty$ is not uniform in $n$, cf. Eq. (4.4). As a consequence, the difference $F^{c}-F$ does not become small, but nevertheless $e^{\Lambda t}\left(F^{c}-F\right)$ does, if $t>0$ :

\section{Lemma 4.8.}

$$
\sup _{t \geqq c^{-1 / 2}} \sup _{\substack{A \in B_{2 R} \\ A \neq 0}}\left(e^{-\lambda t} \frac{\left\|e^{\Lambda t}\left(F^{c}(A)-F(A)\right)\right\|}{\|A\|}\right)=\mathcal{O}\left(\frac{1}{c^{1 / 2}}\right) \text {, as } c \rightarrow \infty \text {. }
$$


Accordingly, setting $A_{1}^{c s}=\Lambda^{c}, A_{2}^{c s}=\Lambda, f_{1}^{c s}=-F^{c}, f_{2}^{c s}=-F$, we see that the assumption (A.5) of Theorem A.2 is fulfilled with $\delta=c^{-1 / 4}$ and $\varepsilon=C c^{-1 / 4}$ for some $C>0$. In view of Eq. (A.6), it follows that $\left\|\Phi_{\eta}^{c}-\Phi_{\eta}\right\|_{\infty}=e^{\beta \eta} \mathcal{O}\left(c^{-1 / 4}\right)$ in $\mathscr{B}_{R} \subset \mathscr{H}$, for all $\eta \geqq c^{-1 / 4}$. Combining this with the fact that $\Phi^{c}, \Phi \in \mathscr{K}_{\beta}$ and choosing $K_{3} \geqq \beta$ sufficiently large, we obtain Eq. (4.7).

We next study the behavior of the semiflow $\Phi_{\eta}^{c}$ around the circles of fixed points corresponding to Eq. (2.4):

$$
\begin{aligned}
& \left.\mathrm{F}_{2}^{c}\right) A_{0}=\frac{-\lambda_{0-}}{\lambda_{0+}-\lambda_{0-}} \sqrt{\alpha_{0}} e^{i \varphi}, \quad A_{n}=0 \text { for all } n \neq 0, \quad \varphi \in \mathbf{R}, \\
& \left.\mathrm{F}_{3}^{c}\right) A_{1}=\frac{-\lambda_{1}-}{\lambda_{1+}-\lambda_{1}-} \sqrt{\alpha_{1}} e^{i \psi}, \quad A_{n}=0 \text { for all } n \neq 1, \quad \psi \in \mathbf{R} .
\end{aligned}
$$

Using Eqs. (4.1), (4.3), it is easy to see that $\mathrm{F}_{2}^{c}, \mathrm{~F}_{3}^{c}$ are close to $\mathrm{F}_{2}, \mathrm{~F}_{3}$ (Sect. 4.2), in the sense that $\operatorname{dist}\left(\mathrm{F}_{2}^{c}, \mathrm{~F}_{2}\right)$ and $\operatorname{dist}\left(\mathrm{F}_{3}^{c}, \mathrm{~F}_{3}\right)$ are $\mathcal{O}\left(1 / c^{2}\right)$ as $c \rightarrow \infty$. Now, let $\hat{A}^{c}$ be the point of $\mathrm{F}_{2}^{c}$ corresponding to $\varphi=0$ in the expression above, and let $A=\hat{A}^{c}+X$, with $X \in \mathscr{B}_{2 \rho} \subset \mathscr{H}$ for some small $\rho>0$. In order to study the evolution of $X$, we introduce the (real) subspaces $V_{s}=\left\{X \in \mathscr{H} \mid \operatorname{Im} X_{0}=0\right\}$, $V_{c}=\left\{X \in \mathscr{H} \mid \operatorname{Re} X_{0}=0, X_{n}=0 \forall n \neq 0\right\}$ and we write $X$ as a pair $\left(X_{s}, X_{c}\right)$ with $X_{s} \in V_{s}, X_{c} \in V_{c}$. With these notations, we have the following result:

Lemma 4.9. If $A=\hat{A}^{c}+X$ is a solution of (4.5') and if $c$ is sufficiently large, then $X=\left(X_{s}, X_{c}\right)$ verifies the differential equation

$$
X_{s}^{\prime}=M_{s}^{c} X_{s}+R_{s}^{c}(X), \quad X_{c}^{\prime}=M_{c}^{c} X_{c}+R_{c}^{c}(X),
$$

where $M_{c}^{c}=0, M_{s}^{c}: V_{s} \rightarrow V_{s}$ is a linear operator satisfying $\left\|e^{M_{s}^{c} t}\right\| \leqq \hat{D} e^{-\frac{1}{2} \alpha_{0} t}$ $\left(t \in \mathbf{R}_{+}\right)$for some $\hat{D} \geqq 1$, and $R^{c}=\left(R_{s}^{c}, R_{c}^{c}\right): \mathscr{H} \rightarrow \mathscr{H}$ is a $\mathscr{C}^{1,1}$ function, vanishing at the origin, such that $\operatorname{Lip}\left(R^{c}\right) \leqq K_{5}\left(\rho+c_{0} / c\right)$ in $\mathscr{B}_{2 \rho} \subset \mathscr{H}$, for some $K_{5}>0$.

It is clear from the proof (see Appendix C) that Lemma 4.9 remains true if $c=\infty$, that is, for the system $\left(4.6^{\prime}\right)$. Indeed, if $\hat{A} \in \mathrm{F}_{2}$ verifies $\hat{A}_{0}=\sqrt{\alpha_{0}}$ and if $A=\hat{A}+X$, then Eq. $\left(4.6^{\prime}\right)$ for $X$ is simply

$$
X_{n}^{\prime}=\left(\alpha_{n}-2 \alpha_{0}\right) X_{n}-\alpha_{0} X_{-n}^{*}+R_{n}(X),
$$

which can be rewritten as

$$
X_{s}^{\prime}=M_{s} X_{s}+R_{s}(X), \quad X_{c}^{\prime}=M_{c} X_{c}+R_{c}(X),
$$

with $M_{s}, M_{c}$ and $R_{s}, R_{c}$ as in Lemma 4.9 .

So, setting $\mathscr{E}^{c s}=V_{s}, \mathscr{E}^{u}=V_{c}, \lambda^{c s}=-\frac{1}{2} \alpha_{0}, \lambda^{u}=0, D=\hat{D}, \ell^{c s}(2 \rho)=\ell^{u}(2 \rho)$ $\leqq K_{5}\left(\rho+c_{0} / c\right)$, and $\beta=-\frac{1}{4} \alpha_{0}$, we can apply Theorem A.1 to both systems (4.8), (4.10), the condition (A.3) being fulfilled if $\rho$ is sufficiently small and if $c_{0} / c \leqq \rho$. Thus, there exist $\mathscr{C}^{1,1}$ maps $h^{c}, h: \mathscr{B}_{\rho} \subset V_{s} \rightarrow V_{c}$, whose graphs $\mathscr{V}^{c}, \mathscr{V}$ are (except for a translation) the local stable manifolds of $\hat{A}^{c}, \hat{A}$ for the semiflows $\Phi_{\eta}^{c}, \Phi_{\eta}$ respectively. In particular, using the phase covariance of the system, we easily obtain the analogue of Lemma 3.8:

Lemma 4.10. There exists a $\rho_{2}>0$ such that, for all sufficiently large c (including $c=\infty)$, the annulus $\mathscr{A}^{c}$ of radius $\rho_{2}$ around the circle $\mathrm{F}_{2}^{c}$ is attracted to $\mathrm{F}_{2}^{c}$ by the semiflow $\Phi_{\eta}^{c}$. 
Moreover, in the same way as Lemma 4.7 and Lemma 4.8, one can prove the following continuity result for $M_{s}^{c}, M_{c}^{c}$ and $R_{s}^{c}, R_{c}^{c}$ :

Lemma 4.11. As $c \rightarrow \infty$,

i) $\sup _{t \geqq c^{-1 / 4}}\left(e^{\frac{1}{2} \alpha_{0} t}\left\|e^{M_{s}^{c} t}-e^{M_{s} t}\right\|\right)=\mathcal{O}\left(\frac{1}{c^{1 / 4}}\right)$,

ii) $\sup _{t \geqq c^{-1 / 2}} \sup _{\substack{X \in \mathscr{B}_{2 \rho} \\ X \neq 0}}\left(e^{\frac{1}{2} \alpha_{0} t} \frac{\left\|e^{M_{s} t}\left(R_{s}^{c}(X)-R_{s}(X)\right)\right\|}{\|X\|}\right)=\mathcal{O}\left(\frac{1}{c^{1 / 2}}\right)$,

iii) $\operatorname{Lip}\left(R_{c}^{c}-R_{c}\right)=\mathcal{O}(1 / c)$.

So, setting $A_{1}^{c s}=M_{s}^{c}, A_{2}^{c s}=M_{s}, A_{1}^{u}=M_{c}^{c}=0, A_{2}^{u}=M_{c}=0, f_{1}^{c s}=R_{s}^{c}, f_{2}^{c s}=R_{s}$, $f_{1}^{u}=R_{c}^{c}, f_{2}^{u}=R_{c}$ and using Lemma 4.11, we see that the assumption (A.5) of Theorem A.2 is fulfilled with $\delta=c^{-1 / 4}$ and $\varepsilon=C c^{-1 / 4}$ for some $C>0$. Thus, it follows from Eq. (A.6) that the unstable manifold $\mathscr{V}^{c}$ is continuous in $c$ at $c=\infty$ in the sense that $\left\|h^{c}-h\right\|_{\infty}=\mathcal{O}\left(c^{-1 / 4}\right)$ in $\mathscr{B}_{\rho_{2}} \subset V_{s}$.

These considerations about the behavior of $\Phi_{\eta}^{c}$ near $\mathrm{F}_{2}^{c}$ can be repeated in an analogous way for $\mathrm{F}_{3}^{c}$; for brevity, we only point out the main differences, and leave the details to the reader. For example, we have instead of Eq. (4.9),

$$
X_{n}^{\prime}=\left(\alpha_{n}-2 \alpha_{1}\right) X_{n}-\alpha_{1} X_{2-n}^{*}+S_{n}(X),
$$

where $S: \mathscr{H} \rightarrow \mathscr{H}$ has the same properties as $R^{c}$ in Lemma 4.9. The spectrum of the linear operator in Eq. (4.11) is contained in the half-plane $\operatorname{Re}(z) \leqq 0$, except for a single positive eigenvalue $\hat{\mu} \geqq \alpha_{0}-2 \varepsilon^{2}$, whose eigenvector $\hat{X}$ is "nearly tangent to the 0-direction" (cf. Sect. 4.2) in the sense that $\hat{X}_{0}=1, \hat{X}_{1}=\mathcal{O}\left(\varepsilon^{2}\right)$ and $\hat{X}_{n}=0$ for all $n \neq 0,2$. So, defining the subspaces $W_{u}=\mathbf{C} \hat{X}, W_{c s}=W_{u}^{\perp}$, we can apply Theorem A.1 and obtain the existence of a local one-dimensional unstable manifold $\mathscr{W}$ as the graph of a $\mathscr{C}^{1,1}$ map $g: \mathscr{B}_{\rho_{3}} \subset W_{u} \rightarrow W_{c s}$, for some (sufficiently small) $\rho_{3}>0$. The same is true for the full system $(c<\infty)$, and the manifold $\mathscr{W}^{c}$ depends continuously on $c$ in the sense that $\operatorname{dist}\left(\mathscr{W}^{c}, \mathscr{W}\right)=\mathcal{O}\left(c^{-1 / 4}\right)$ as $c \rightarrow \infty$.

Now, let us summarize our results. The semiflow $\Phi_{\eta}^{c}$ corresponding to (4.5') is continuous in $c$ at $c=\infty$ (in the sense of Proposition 4.6), and so are the fixed points $\mathrm{F}_{2}^{c}, \mathrm{~F}_{3}^{c}$. Moreover, the circle $\mathrm{F}_{2}^{c}$ has an attractive annular neighborhood $\mathscr{A}^{c}$, of radius $\rho_{2}$ independent of $c$ (Lemma 4.10), and at each point of $\mathrm{F}_{3}^{c}$ one can attach a local one-dimensional unstable manifold $\mathscr{W}^{c}$ which depends continuously on $c$ at $c=\infty$. Combining these facts with Proposition 4.5 in the same way as in Sect. 3.4, we see that there exists a $c_{1} \geqq c_{0}$ such that, for all $c \geqq c_{1}$ and all $\varepsilon \leqq \varepsilon_{1}$, the continuation of the local unstable manifold $\mathscr{W}^{c}$ intersects the attractive neighborhood $\mathscr{A}^{c}$ of $F_{2}^{c}$. This concludes the proof of Theorem 1.2.

\section{Appendix A. The Center-Stable Manifold Theorem}

In this appendix, we recall (for easy reference) some results of center manifold theory in infinite-dimensional Banach spaces. Proofs of these statements can be found in the companion paper by one of us [G]. They are an extension of results of [EW], Appendix A. 
Let $\left(\mathscr{E}^{c s},\|\cdot\|_{c s}\right),\left(\mathscr{E}^{u},\|\cdot\|_{u}\right)$ be two Banach spaces, and denote by $\mathscr{E}$ the direct sum $\mathscr{E}^{\text {cs }} \oplus \mathscr{E}^{u}$ equipped with the norm $\|z\|=\max \left(\left\|z^{c s}\right\|_{c s},\left\|z^{u}\right\|_{u}\right)$, for $z=\left(z^{c s}, z^{u}\right) \in \mathscr{E}$. We consider the differential equation in $\mathscr{E}$,

$$
\begin{gathered}
\frac{d z^{c s}}{d t}=A^{c s} z^{c s}+f^{c s}\left(z^{c s}, z^{u}\right), \\
\frac{d z^{u}}{d t}=A^{u} z^{u}+f^{u}\left(z^{c s}, z^{u}\right),
\end{gathered}
$$

and make the following hypotheses:

H1) The linear operators $A^{c s}: \mathscr{E}^{c s} \rightarrow \mathscr{E}^{c s}$ and $-A^{u}: \mathscr{E}^{u} \rightarrow \mathscr{E}^{u}$ define strongly continuous semigroups $e^{A^{c s t}}, e^{-A^{u t}}$ for $t \geqq 0$. Moreover, there exist real constants $\lambda^{c s}, \lambda^{u}, D$ such that $\lambda^{u} \geqq 0, \lambda^{u}>\max \left(\lambda^{s}, 2 \lambda^{s}\right)$, and

$$
\left\|e^{A^{c s t}}\right\|_{c s} \leqq D e^{\lambda c s_{t}}, \quad\left\|e^{-A^{u_{t}}}\right\|_{u} \leqq D e^{-\lambda^{u_{t}}}
$$

for all $t \geqq 0$.

H2) $f^{c s}: \mathscr{E} \rightarrow \mathscr{E}^{c s}$ and $f^{u}: \mathscr{E} \rightarrow \mathscr{E}^{u}$ are $\mathscr{C}^{1,1}$ functions vanishing at the origin together with their first derivatives.

H3) The norm $\|\cdot\|_{c s}$ is a $\mathscr{C}^{1,1}$ function on $\mathscr{E}^{c s}$.

Under these hypotheses, the center-stable manifold theorem asserts the existence, in a small neighborhood of the fixed point 0 , of a $\mathscr{C}^{1,1}$ manifold $\Gamma$ which is tangent to the subspace $\mathscr{E}^{\text {cs }}$ at the origin, is left invariant by Eq. (A.1) and contains all the trajectories which stay near 0 for all $t \in \mathbf{R}_{+}$. We shall give here an explicit formulation of this theorem, because in our applications to the system (2.3), we need to know exactly how the manifold depends on the parameters $\varepsilon$ and $c$.

In order to do that, we introduce some more notations. For all $r>0$, we denote by $\mathscr{B}_{r}^{c s}, \mathscr{B}_{r}^{u}, \mathscr{B}_{r}$ the balls of radius $r$ around the origin in $\mathscr{E}^{c s}, \mathscr{E}^{u}, \mathscr{E}$ respectively, and we set

$$
\ell^{c s}(r)=\sup _{z \in \mathscr{B}_{r}}\left\|D f^{c s}(z)\right\|, \quad \ell^{u}(r)=\sup _{z \in \mathscr{B}_{r}}\left\|D f^{u}(z)\right\|,
$$

where $D f^{c s}, D f^{u}$ are the derivatives of $f^{c s}, f^{u}$. In view of $\mathrm{H} 2, \ell^{c s}(r), \ell^{u}(r) \rightarrow 0$ as $r \rightarrow 0$. Next, for all $\sigma \in[0,1], \beta \in\left(\lambda^{c s}, \lambda^{u}\right)$, we define the function spaces

$$
\begin{aligned}
H_{\sigma}= & \left\{h: \mathscr{E}^{c s} \rightarrow \mathscr{E}^{u} \mid h(0)=0 ;\|h(\xi)-h(\tilde{\xi})\|_{u} \leqq \sigma\|\xi-\tilde{\xi}\|_{c s} \forall \xi, \tilde{\xi} \in \mathscr{E}^{c s}\right\}, \\
\mathscr{K}_{\beta}=\{ & \left\{\Phi: \mathbf{R}_{+} \times \mathscr{E}^{c s} \rightarrow \mathscr{E}^{c s} \mid \Phi_{0}(\xi)=\xi \forall \xi \in \mathscr{E} c\right. \\
& \Phi_{t}(0)=0 \forall t \in \mathbf{R}_{+} ; \Phi \text { is continuous in } t ; \\
& \left.\left\|\Phi_{t}(\xi)-\Phi_{t}(\tilde{\xi})\right\|_{c s} \leqq D e^{\beta t}\|\xi-\tilde{\xi}\|_{c s} \forall t \in \mathbf{R}_{+}, \forall \xi, \tilde{\xi} \in \mathscr{E} c s\right\} .
\end{aligned}
$$

Finally, defining $\hat{\beta}=\max (\beta, 2 \beta)$, we know from H1 that there exists a $\beta \in\left(\lambda^{c s}, \lambda^{u}\right)$ such that $\hat{\beta} \in\left(\lambda^{c s}, \lambda^{u}\right)$. For such a $\beta$, we set

$$
\sigma=\sigma(r, \beta)=D^{2} \max \left(\frac{5 \ell^{c s}(2 r)}{\beta-\lambda^{c s}}, \frac{5 \ell^{u}(2 r)}{\lambda^{u}-\hat{\beta}}\right) .
$$

With these notations, we have the following result: 
Theorem A.1. [G]. Assume that the hypotheses H1, H2, H3, are fulfilled, and choose $r$ so small that, in Eq. (A.2),

$$
\sigma<\frac{1}{2 D}
$$

Then there exist a map $h \in H_{\sigma}$ and a semiflow $\Phi \in \mathscr{K}_{\beta}$ with the following properties:

i) $h$ is of class $\mathscr{C}^{1,1}, D h(0)=0$, and $h$ maps $\mathscr{D}\left(A^{c s}\right)$ (the domain of $\left.A^{c s}\right)$ into $\mathscr{D}\left(A^{u}\right)$ (the domain of $A^{u}$ ).

ii) For all $\xi \in \mathscr{B}_{r}^{c s} \cap \mathscr{D}\left(A^{c s}\right)$, the curve $z(t)=\left(\Phi_{t}(\xi), h\left(\Phi_{t}(\xi)\right)\right), t \in \mathbf{R}_{+}$, is a solution of Eq. (A.1) as long as it remains in $\mathscr{B}_{r}$.

iii) If $z(t)$ is any solution of $E q$. (A.1) such that $z(t) \in \mathscr{B}_{r}$ for all $t \in \mathbf{R}_{+}$, then $z(t)=\left(\Phi_{t}(\xi), h\left(\Phi_{t}(\xi)\right)\right)$ for some $\xi \in \mathscr{B}_{r}^{c s}$.

Thus, denoting by $\Gamma_{r}$ the restriction of the graph of $h$ to $\mathscr{B}_{r}$, we see that Eq. (A.1) defines a local semiflow on $\Gamma_{r}$ (in the sense of ii). We shall always refer to $\Gamma_{r}$ as the (local) center-stable manifold, although in the case $\lambda^{c s}<0$ one rather speaks of a stable manifold.

\section{Remarks.}

1) In the proof of Theorem A.1, one has to "cut off" the non-linear terms $f^{c s}, f^{u}$ outside the ball $\mathscr{B}_{r}^{c s}$. Since (by H3) the norm $\|\cdot\|_{c s}$ is $\mathscr{C}^{1,1}$ on $\mathscr{E} c$, this is simply done by writing $f\left(z^{c s}, z^{u}\right) \chi\left(\left\|z^{c s}\right\|_{c s} / r\right)$, where $\chi: \mathbf{R}_{+} \rightarrow[0,1]$ is some $\mathscr{C}^{1,1}$ function equal to 1 on $[0,1]$, vanishing on $[2, \infty)$, and satisfying $\left|\chi^{\prime}(x)\right| \leqq 2$ for all $x \in \mathbf{R}_{+}$. This is how the expressions $5 \ell^{c s}(2 r), 5 \ell^{u}(2 r)$ arise in Eq. (A.2). For more details, see [G], Sect. 3.2.

2) Except for the assertion $D h(0)=0$, Theorem A.1 remains true if $D f^{c s}, D f^{u}$ are not assumed to vanish at origin, provided that Eq. (A.3) can be satisfied for $r$ sufficiently small.

3) As a consequence of iii), all fixed points of the system (A.1) in $\mathscr{B}_{r}$ must lie on the center-stable manifold $\Gamma_{r}$.

4) If the non-linear term $f=\left(f^{c s}, f^{u}\right)$ commutes with a linear isometry of $\mathscr{E}$, then $h$ can be chosen to commute with the same isometry.

5) Theorem A.1 also makes sense if $\mathscr{E}^{\mathscr{c s}}=\mathscr{E}$ and $\mathscr{E}^{u}=\{0\}$. In this case, it only asserts that the solutions of the system

$$
\frac{d z^{c s}}{d t}=A^{c s} z^{c s}+f^{c s}\left(z^{c s}\right)
$$

define a local semiflow $\Phi \in \mathscr{K}_{\beta}$ in the ball $\mathscr{B}_{r}$ around the origin. Setting $\lambda^{u}=+\infty, \ell^{u}(2 r)=0$, the condition (A.2), (A.3) reduces to

$$
\sigma=D^{2} \frac{5 \ell^{c s}(2 r)}{\beta-\lambda^{c s}}<\frac{1}{2 D}
$$

which is always satisfied if $\beta>\lambda^{c s}$ is sufficiently large.

It is well-known that the center-stable manifold, although generally not unique, can be chosen to depend continuously (for suitable topologies) on the operators $A^{c s}, A^{u}$ and the functions $f^{c s}, f^{u}$ in Eq. (A.1). We shall give here a formulation of this result which is sufficient for our applications in Sect. 3 and Sect. 4. Suppose that we are given two pairs of linear operators $A_{1}^{c s}, A_{1}^{u}$ and $A_{2}^{c s}, A_{2}^{u}$ satisfying $\mathrm{H} 1$ with the 
same constants $\lambda^{c s}, \lambda^{u}, D$. Assume also that we have two couples of functions $f_{1}^{c s}, f_{1}^{u}$ and $f_{2}^{c s}, f_{2}^{u}$ verifying $\mathrm{H} 2$, and that the condition (A.3) is fulfilled for both systems 1 and 2 . Denoting thus by $h_{1}, h_{2}$ and $\Phi_{1}, \Phi_{2}$ the maps and semiflows whose existence is asserted by Theorem A.1, we have the following result:

Theorem A.2. Assume that there exist an $\varepsilon>0$ and $a \delta \geqq 0$ such that

$$
\begin{aligned}
& \sup _{t \geqq \delta}\left(e^{-\lambda c s t}\left\|e^{A_{1}^{c s} t}-e^{A_{2}^{c s} t}\right\|_{c s}\right) \leqq D \varepsilon, \\
& \sup _{t \geq \delta}\left(e^{\lambda^{u} t}\left\|e^{-A_{1}^{u} t}-e^{-A_{2}^{u} t}\right\|_{u}\right) \leqq D \varepsilon,
\end{aligned}
$$

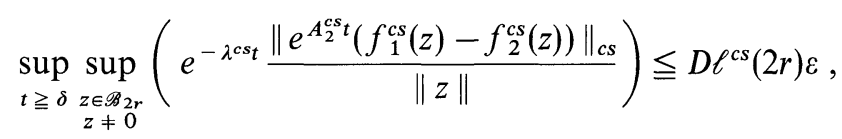

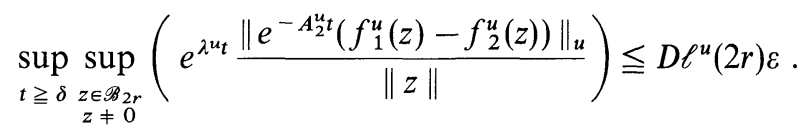

Then $h_{1}, h_{2}$ and $\Phi_{1}, \Phi_{2}$ can be chosen so that

$$
\begin{array}{r}
\sup _{\xi \neq 0} \frac{\left\|h_{1}(\xi)-h_{2}(\xi)\right\|_{u}}{\|\xi\|_{c s}} \leqq \frac{1}{1-2 \sigma}\left(D \varepsilon+3 \delta\left(\lambda^{u}-\lambda^{c s}\right)\right), \\
\sup _{t \geqq \delta \xi \neq 0}\left(e^{-\beta t} \frac{\left\|\Phi_{1, t}(\xi)-\Phi_{2, t}(\xi)\right\|_{c s}}{\|\xi\|_{c s}}\right) \leqq \frac{1}{1-2 \sigma}\left(D \varepsilon+3 \delta\left(\lambda^{u}-\lambda^{c s}\right)\right) .
\end{array}
$$

For a proof in the case $\delta=0$, see [G], Sect. 2.3. The general case is easily proved along the same lines.

\section{Appendix B. Some Proofs (Unstable-Unstable case)}

Proof of Lemma 3.7. We first write the vector field $\Delta \chi(x)$ in the form

$$
\begin{aligned}
& \Delta \chi_{0}(x)=\left(-\frac{\lambda_{0+}}{\varepsilon}-\frac{2}{c-2 i}\right) X_{0}-\frac{\Delta_{0}(x)}{c-2 i}+\left(\frac{-1}{\lambda_{0+}-\lambda_{0-}}+\frac{1}{c-2 i}\right) F_{0}(x+l(x)), \\
& \Delta \chi_{1}(x)=\left(-\frac{\lambda_{1}+}{\varepsilon}-\frac{2 \alpha}{c+2 i}\right) X_{1}-\frac{\Delta_{1}(x)}{c+2 i}+\left(\frac{-1}{\lambda_{1+}-\lambda_{1-}}+\frac{1}{c+2 i}\right) F_{1}(x+l(x)),
\end{aligned}
$$

where $\Delta: \mathscr{B}_{2 R} \subset \mathscr{H}_{0} \rightarrow \mathscr{H}$ is defined by $\Delta(x)=F(x+l(x))-F(x)$. Next, we use the identity

$$
F(A+B)=F(A)+G(A, B)+G(B, A)+F(B), \quad A, B \in \mathscr{H},
$$

where $G: \mathscr{H} \times \mathscr{H} \rightarrow \mathscr{H}$ is the Fréchet derivative of $F$ :

$$
G_{n}(A, B)=\sum_{p+s+r=n}\left(A_{p} A_{s} B_{-r}^{*}+2 A_{p} B_{s} A_{-r}^{*}\right)
$$


As is easily seen,

$$
\|G(A, B)-G(\tilde{A}, \tilde{B})\| \leqq 3 K^{2}\left(\|A-\tilde{A}\|(\|A\|+\|\tilde{A}\|)\|B\|+\|\tilde{A}\|^{2}\|B-\tilde{B}\|\right),
$$

with $K$ as in (2.5). Now, using (B.1) with $A=x$ and $B=l(x)$, we can write $\Delta(x)=G(x, l(x))+G(l(x), x)+F(l(x))$. Using (B.3) and recalling that $\operatorname{Lip}(l) \leqq \ell \varepsilon / \varepsilon_{c}$, we thus obtain

$$
\begin{aligned}
\operatorname{Lip}(\Delta) & \leqq \operatorname{Lip}(G(\cdot, l(\cdot)))+\operatorname{Lip}(G(l(\cdot), \cdot))+\operatorname{Lip}(F(l(\cdot))) \\
& \leqq C \varepsilon / \varepsilon_{c}+C\left(\varepsilon / \varepsilon_{c}\right)^{2}+C\left(\varepsilon / \varepsilon_{c}\right)^{3} \leqq 3 C \varepsilon / \varepsilon_{c}
\end{aligned}
$$

in $\mathscr{B}_{2 R}$, for some $C>0$. In the same way, it follows from (2.5) that $\operatorname{Lip}(F(\cdot+l(\cdot))) \leqq 3 K(2 R)^{2}(1+\ell)^{2}$ in $\mathscr{B}_{2 R}$. Finally, in view of (3.1), (3.4), we have

$$
\left|-\frac{\lambda_{0+}}{\varepsilon}-\frac{2}{c-2 i}\right|=\mathcal{O}\left(\frac{\varepsilon}{c+2}\right),\left|\frac{-1}{\lambda_{0+}-\lambda_{0-}}+\frac{1}{c-2 i}\right|=\mathcal{O}\left(\frac{\varepsilon}{(c+2)^{2}}\right),
$$

and similarly for $\lambda_{1+}, \lambda_{1+}$. Combining these estimates, we easily find

$$
\operatorname{Lip}(\Delta \chi) \leqq K_{4}\left(\varepsilon / \varepsilon_{c}\right) /(c+2)
$$

in $\mathscr{B}_{2 R}$, for some $K_{4}>0$.

Proof of Lemma 3.9. Using the definitions of $\delta \chi$ and $\Delta \chi$, we easily obtain the identity

$$
\delta \chi(z)-\delta \chi(\tilde{z})=\Delta \chi\left(x^{\varepsilon}+z\right)-\Delta \chi\left(x^{\varepsilon}+\tilde{z}\right)+\Delta_{\varepsilon}(z, \tilde{z}),
$$

where $\Delta_{\varepsilon}(z, \tilde{z})=\left(\chi^{0}\left(x^{\varepsilon}+z\right)-\chi^{0}\left(x^{0}+z\right)\right)-\left(\chi^{0}\left(x^{\varepsilon}+\tilde{z}\right)-\chi^{0}\left(x^{0}+\tilde{z}\right)\right)$. Since $x^{\varepsilon} \in \mathscr{B}_{\boldsymbol{R}}$ $\subset \mathscr{H}_{0}$, it follows from Lemma 3.7 that

$$
\left\|\Delta \chi\left(x^{\varepsilon}+z\right)-\Delta \chi\left(x^{\varepsilon}+\tilde{z}\right)\right\| \leqq \frac{K_{4}}{c+2} \frac{\varepsilon}{\varepsilon_{c}}\|z-\tilde{z}\|
$$

for all $z, \tilde{z} \in \mathscr{B}_{R}$; so, it remains to bound the function $\Delta_{\varepsilon}(z, \tilde{z})$. First, if $D \chi^{0}$ denotes the derivative of $\chi^{0}$, it is easy to see that $\operatorname{Lip}\left(D \chi^{0}\right) \leqq C_{1} /(c+2)$ in $\mathscr{B}_{2 R} \subset \mathscr{H}_{0}$, for some $C_{1}>0$. Next, since $\lambda_{0 \pm}$ and $\sqrt{2-\varepsilon}$ are smooth functions of $\varepsilon$, the curve $\varepsilon \rightarrow x^{\varepsilon}$ is $\mathscr{C}^{1}$ and $\left\|\left(d x^{\varepsilon} / d \varepsilon\right)\right\| \leqq C_{2}$ for some $C_{2}>0$. So, $\Delta_{\varepsilon}(z, \tilde{z})$ is differentiable in $\varepsilon$ and

$$
\left\|\frac{\partial \Delta_{\varepsilon}(z, \tilde{z})}{\partial \varepsilon}\right\|=\left\|\left(D \chi^{0}\left(x^{\varepsilon}+z\right)-D \chi^{0}\left(x^{\varepsilon}+\tilde{z}\right)\right) \cdot \frac{d x^{\varepsilon}}{d \varepsilon}\right\| \leqq \frac{C_{1}}{c+2}\|z-\tilde{z}\| \cdot C_{2} .
$$

Since $\Delta_{0}(z, \tilde{z})=0$, we find

$$
\left\|\Delta_{\varepsilon}(z, \tilde{z})\right\| \leqq \varepsilon \sup _{\varepsilon}\left\|\frac{\partial \Delta_{\varepsilon}(z, \tilde{z})}{\partial \varepsilon}\right\| \leqq \frac{C_{1} C_{2} \varepsilon}{c+2}\|z-\tilde{z}\| \leqq \frac{K_{0} C_{1} C_{2}}{c+2} \frac{\varepsilon}{\varepsilon_{c}}\|z-\tilde{z}\| .
$$

This concludes the proof of Lemma 3.9, with $K_{6}=K_{4}+K_{0} C_{1} C_{2}$. 


\section{Appendix C. Some Proofs (Stable-Unstable Case)}

Proof of Lemma 4.2. To simplify the notation, we set $A_{\|}=a$ and $A_{\perp}=X$. Inserting $A=a+X$ in (4.6), we obtain for $|n| \geqq 2$ :

$$
X_{n}^{\prime}=-F_{n}(a)+\alpha_{n} X_{n}-G_{n}(a, X)-G_{n}(X, a)-F_{n}(X),
$$

where $G$ is the derivative of $F$ (cf. (B.1), (B.2)). We want to obtain an upper bound on the quantity $\frac{1}{2} \frac{d}{d \eta}\|X\|^{2}=\operatorname{Re}\left(X, X^{\prime}\right)$. First of all, using the definition (1.7), we find $F_{2}(a)=a_{1}^{2} a_{0}^{*}+2 a_{1} a_{0} a_{-}^{*}, F_{3}(a)=a_{1}^{2} a_{-1}^{*}, F_{n}(a)=0$ for all $n>3$, and symmetric expressions hold for $n<0$. If $a \in D_{\varepsilon}$, we thus have

$$
|\operatorname{Re}(X, F(a))| \leqq\|X\| C_{1} \varepsilon^{2},
$$

for some $C_{1}>0$. Let us now consider the linear terms in (C.1). Since $\alpha_{n} \leqq \alpha_{2} \leqq-3+4 \varepsilon^{2}<0$ for all $|n| \geqq 2$, we have $\operatorname{Re}(X, \alpha X) \leqq$ $\|X\|^{2}\left(-3+\mathcal{O}\left(\varepsilon^{2}\right)\right)$. On the other hand, a direct calculation yields $G_{n}(a, X)=2 X_{n}\left|a_{0}\right|^{2}+\hat{G}_{n}(a, X)$, where $\hat{G}_{n}(a, X)$ is some complicated expression satisfying $\|\hat{G}(a, X)\| \leqq\|X\|\left(\left|a_{0}\right|^{2}+\mathcal{O}(\varepsilon)\right)$ if $a \in D_{\varepsilon}$. As a consequence, $\operatorname{Re}(X,-G(a, X)) \leqq\|X\|^{2} \mathcal{O}(\varepsilon)$. Combining these estimates, we obtain for the linear terms

$$
\operatorname{Re}(X, \alpha X-G(a, X)) \leqq\|X\|^{2}(-3+\mathcal{O}(\varepsilon)) .
$$

Finally, we just bound the two remaining terms in (C.1) by using (2.5), (B.3); we find

$$
|\operatorname{Re}(X, G(X, a))| \leqq\|X\|^{3} C_{2}, \quad|\operatorname{Re}(X, F(X))| \leqq\|X\|^{4} K^{2},
$$

for some $C_{2}>0$. Now, we summarize (C.2)-(C.4)

$$
\frac{1}{2} \frac{d}{d \eta}\|X\|^{2} \leqq\|X\| C_{1} \varepsilon^{2}+\|X\|^{2}(-3+\mathcal{O}(\varepsilon))+\|X\|^{3} C_{2}+\|X\|^{4} K^{2},
$$

and choose a $K_{2}>0$ such that $3 K_{2}>C_{1}$. Then, assuming that $\varepsilon$ is sufficiently small, it is easy to verify that the right-hand side of (C.5) is negative if $\|X\|=K_{2} \varepsilon^{2}$.

Proof of Lemma 4.3. Let $A=a+X$ with $\|X\| \leqq K_{2} \varepsilon^{2}, a \in D_{\varepsilon},\left|a_{1}\right|=2 \varepsilon$. Inserting in (4.6), we obtain for $n=1$ :

$$
a_{1}^{\prime}=\alpha_{1} a_{1}-F_{1}(a)-G_{1}(a, X)-G_{1}(X, a)-F_{1}(X) .
$$

Again, we want an upper bound on $\frac{1}{2} \frac{d}{d \eta}\left|a_{1}\right|^{2}=\operatorname{Re}\left(a_{1}^{*} a_{1}^{\prime}\right)$. First of all, $F_{1}(a)=$ $a_{1}\left(\left|a_{1}\right|^{2}+2\left|a_{0}\right|^{2}+2\left|a_{-1}\right|^{2}\right)+a_{0}^{2} a_{-1}^{*}$, and since $\operatorname{Re}\left(-\left|a_{1}\right|^{2}\left|a_{0}\right|^{2}+a_{1}^{*} a_{0}^{2} a_{-1}^{*}\right)$ $\leqq 0$, we have

$$
\operatorname{Re}\left(\alpha_{1}\left|a_{1}\right|^{2}-a_{1}^{*} F_{1}(a)\right) \leqq\left|a_{1}\right|^{2}\left(\alpha_{1}-\left|a_{1}\right|^{2}-\left|a_{0}\right|^{2}\right) .
$$

On the other hand, a direct calculation yields $G_{1}(a, X)=a_{-1}^{2} X_{-3}^{*}+2 a_{0} a_{-1} X_{-2}^{*}$ $+2 a_{0} a_{1}^{*} X_{2}+2 a_{0}^{*} a_{-1} X_{2}+2 a_{1}^{*} a_{-1} X_{3}$, so that

$$
\left|\operatorname{Re}\left(a_{1}^{*} G_{1}(a, X)\right)\right| \leqq\left|a_{1}\right|\|X\|\left(C_{3}\left|a_{0}\right| \varepsilon+\mathcal{O}\left(\varepsilon^{2}\right)\right),
$$


for some $C_{3}>0$. Finally, the two remaining terms in (C.6) are simply bounded by

$$
\left|\operatorname{Re}\left(a_{1}^{*} G_{1}(X, a)\right)\right| \leqq\left|a_{1}\right|\|X\|^{2} C_{2}, \quad\left|\operatorname{Re}\left(a_{1}^{*} F_{1}(X)\right)\right| \leqq\left|a_{1}\right|\|X\|^{3} K^{2} .
$$

Now, recalling that $\alpha_{1}=\varepsilon^{2},\left|a_{1}\right|=2 \varepsilon,\|X\| \leqq K_{2} \varepsilon^{2}$, we can summarize (C.7)-(C.9) as follows:

$$
\frac{1}{2} \frac{d}{d \eta}\left|a_{1}\right|^{2} \leqq 4 \varepsilon^{2}\left(-3 \varepsilon^{2}-\left|a_{0}\right|^{2}\right)+2 C_{3} K_{2}\left|a_{0}\right| \varepsilon^{4}+\mathcal{O}\left(\varepsilon^{5}\right)
$$

The maximum over $\left|a_{0}\right|$ of the right-hand side (reached for $\left|a_{0}\right|=\frac{1}{4} C_{3} K_{2} \varepsilon^{2}$ ) is of the form $-12 \varepsilon^{4}+\mathcal{O}\left(\varepsilon^{5}\right)$, and thus is negative if $\varepsilon$ is sufficiently small.

Proof of Lemma 4.4. As before, let $A=a+X$ with $a \in D_{\varepsilon}$ and $\|X\| \leqq K_{2} \varepsilon^{2}$. Inserting in (4.6), we obtain for $n=0$ :

$$
a_{0}^{\prime}=\alpha_{0} a_{0}-F_{0}(a)-G_{0}(a, X)-G_{0}(X, a)-F_{0}(X) .
$$

We now want to find a lower bound on the quantity $\frac{1}{2} \frac{d}{d \eta}\left|a_{0}\right|^{2}=\operatorname{Re}\left(a_{0}^{*} a_{0}\right)$. First of all, we have $F_{0}(a)=a_{0}\left(\left|a_{0}\right|^{2}+2\left|a_{1}\right|^{2}+2\left|a_{-1}\right|^{2}\right)+2 a_{0}^{*} a_{1} a_{-1}$, and since $\left|a_{1}\right| \leqq 2 \varepsilon,\left|a_{-1}\right| \leqq 2 \varepsilon$, we can write

$$
\operatorname{Re}\left(\alpha_{0}\left|a_{0}\right|^{2}-a_{0}^{*} F_{0}(a)\right) \geqq\left|a_{0}\right|^{2}\left(\alpha_{0}-\left|a_{0}\right|^{2}-24 \varepsilon^{2}\right) .
$$

On the other hand, we have $G_{0}(a, X)=a_{1}^{2} X_{2}^{*}+a_{-1}^{2} X_{-2}^{*}+2 a_{1} a_{-1}^{*} X_{-2}$ $+2 a_{1}^{*} a_{-1} X_{2}$, and thus

$$
-\operatorname{Re}\left(a_{0}^{*} G_{0}(a, X)\right) \geqq-\left|a_{0}\right|\|X\| C_{4} \varepsilon^{2},
$$

for some $C_{4}>0$. Finally, we find as above

$$
-\operatorname{Re}\left(a_{0}^{*} G_{0}(X, a)\right) \geqq-\left|a_{0}\right|\|X\|^{2} C_{2}, \quad-\operatorname{Re}\left(a_{0}^{*}, F_{0}(X)\right) \geqq-\left|a_{0}\right|\|X\|^{3} K^{2} .
$$

Recalling that $\|X\| \leqq K_{2} \varepsilon^{2}$, we thus obtain

$$
\frac{1}{2} \frac{d}{d \eta}\left|a_{0}\right|^{2} \geqq\left|a_{0}\right|^{2}\left(\alpha_{0}-\left|a_{0}\right|^{2}-24 \varepsilon^{2}\right)-\left|a_{0}\right|\left(K_{2} C_{4} \varepsilon^{4}+\mathcal{O}\left(\varepsilon^{5}\right)\right) .
$$

Assuming that $\varepsilon$ is sufficiently small, it is easy to verify that the right-hand side is positive and bounded away from zero if $\varepsilon^{3} \leqq\left|a_{0}\right| \leqq \sqrt{\alpha_{0}}(1-\varepsilon)$.

Proof of Lemma 4.7. We have to bound the difference $e^{\alpha_{n}(c) t}-e^{\alpha_{n} t}$ for sufficiently large $c \geqq c_{0}$. In view of (4.1), $\alpha_{n}(c)=-c \lambda_{n+}$ is in fact a function of $c$ and $q_{n}$, and we shall deal with the cases $\left|q_{n}\right| \leqq c^{1 / 4},\left|q_{n}\right|>c^{1 / 4}$ separately. First, if $\left|q_{n}\right| \leqq c^{1 / 4}$, we always have $c \geqq 8\left(1+\left|q_{n}\right|\right)$ if $c$ is sufficiently large, so that $\left|\alpha_{n}(c)-\alpha_{n}\right|$ $\leqq(5 / c)\left(1+\left|q_{n}\right|\right)^{3} \leqq 40 / c^{1 / 4}$, by (4.1). Since $\alpha_{n} \leqq 1$ and $\operatorname{Re} \alpha_{n}(c) \leqq c \hat{\lambda}=1+\mathcal{O}\left(1 / c^{2}\right)$ for all $n$, it follows that

$$
\left|e^{\alpha_{n}(c) t}-e^{\alpha_{n} t}\right| \leqq t e^{c \hat{\lambda} t}\left|\alpha_{n}(c)-\alpha_{n}\right| \leqq e^{\lambda t} \sup _{t \geqq 0}\left(t e^{(c \hat{\lambda}-\lambda) t}\right) \frac{40}{c^{1 / 4}}=e^{\lambda t} \mathcal{O}\left(\frac{1}{c^{1 / 4}}\right)
$$


for all $t \geqq 0$. Conversely, assume that $\left|q_{n}\right|>c^{1 / 4}$; then, since $\operatorname{Re} \alpha_{n}(c)$ is a decreasing function of $\left|q_{n}\right|$ (as can be seen from Fig. 4), we have the bound

$$
\begin{aligned}
\operatorname{Re}\left(\alpha_{n}(c)\right) & =\frac{c^{2}}{2} \operatorname{Re}\left(1-\sqrt{1-\frac{4}{c^{2}}+\frac{4 i q_{n}}{c}}\right) \leqq \frac{c^{2}}{2} \operatorname{Re}\left(1-\sqrt{1-\frac{4}{c^{2}}+\frac{4 i}{c^{3 / 4}}}\right) \\
& \leqq \frac{c^{2}}{2}\left(1-\left(1+\frac{2}{c^{3 / 2}}+\mathcal{O}\left(1 / c^{2}\right)\right)\right)=-\sqrt{c}+\mathcal{O}(1) .
\end{aligned}
$$

As a consequence, $\operatorname{Re} \alpha_{n}(c) \leqq-\sqrt{c} / 2$ if $c$ is sufficiently large, and since $\alpha_{n} \leqq 1-\sqrt{c}$, the same is true for $\operatorname{Re} \alpha_{n}$. Thus, for all $t \geqq c^{-1 / 4}$, we have $\left|e^{\alpha_{n}(c) t}-e^{\alpha_{n} t}\right| \leqq 2 e^{-\sqrt{c} t / 2} \leqq 2 e^{-\frac{1}{2} c^{1 / 4}} \leqq 4 / c^{1 / 4}$. Combining the two cases $\left|q_{n}\right| \leqq c^{1 / 4}$, $\left|q_{n}\right|>c^{1 / 4}$, the assertion follows.

Proof of Lemma 4.8. Using the definitions of $\Lambda, F^{c}, F$, we easily obtain for all $t \in \mathbf{R}_{+}$,

$$
\begin{aligned}
\left\|e^{\Lambda t}\left(F^{c}(A)-F(A)\right)\right\| \leqq & \left\|e^{\Lambda t}(F(A+h(A))-F(A))\right\| \\
& +\sup _{n \in \mathbf{Z}}\left(e^{\alpha_{n} t}\left|v_{n}(c)-1\right|\right)\|F(A+h(A))\| .
\end{aligned}
$$

In view of Proposition 4.1, we have $\|F(A+h(A))-F(A)\| \leqq K_{4} \frac{1}{2}\left(c_{0} / c\right)^{2}\|A\|$ and $\|F(A+h(A))\| \leqq K_{4}\left(1+\frac{1}{2}\left(c_{0} / c\right)^{2}\right)\|A\|$ for all $A \in \mathscr{B}_{2 R} \subset \mathscr{H}$. So, it remains to bound the difference $e^{\alpha_{n} t}\left(v_{n}(c)-1\right)$, and this can be done as above, by dealing with the cases $\left|q_{n}\right| \leqq \sqrt{c},\left|q_{n}\right|>\sqrt{c}$ separately. First, if $\left|q_{n}\right| \leqq \sqrt{c}$, we always have $c \geqq 8\left(1+\left|q_{n}\right|\right)$ if $c$ is sufficiently large, and thus $\left|v_{n}(c)-1\right| \leqq(4 / c)\left(1+\left|q_{n}\right|\right)$ $\leqq(8 / c)(1+\sqrt{c}) \leqq(8 / \sqrt{c})$, by (4.4). So, $e^{\alpha_{n} t}\left|v_{n}(c)-1\right| \leqq(8 / \sqrt{c}) e^{\lambda t}$ in this case. Conversely, if $\left|q_{n}\right|>\sqrt{c}$, then $\alpha_{n}=1-q_{n}^{2} \leqq 1-c$, so that $e^{\alpha_{n} t} \leqq e^{\lambda t} e^{-c t} \leqq$ $e^{\lambda t} e^{-\sqrt{c}} \leqq e^{\lambda t} / \sqrt{c}$ for all $t \geqq 1 / \sqrt{c}$; on the other hand, $\left|v_{n}(c)-1\right| \leqq 1+\mathcal{O}(1)$ by (4.3). So, combining the results for $\left|q_{n}\right| \leqq \sqrt{c}$ and $\left|q_{n}\right|>\sqrt{c}$, we obtain

$$
\sup _{t \geqq c^{-1 / 2}} \sup _{n \in \mathbf{Z}}\left(e^{\alpha_{n} t}\left|v_{n}(c)-1\right| e^{-\lambda t}\right) \leqq \frac{C}{\sqrt{c}},
$$

for some $C>0$. Combining this with (C.16), the assertion follows.

Proof of Lemma 4.9. By definition of $\hat{A}^{c}$ and by construction of the center-unstable manifold, we have $\hat{A}^{c}+h\left(\hat{A}^{c}\right)=C \in \mathscr{H}$, where $C_{n}=\sqrt{\alpha_{0}} \delta_{n, 0}$. Thus, if $A=\hat{A}^{c}+X$, then $A+h(A)=C+X+\Delta h(X)$, where $\Delta h(X)=h\left(\hat{A}^{c}+X\right)-h\left(\hat{A}^{c}\right)$. As a consequence, it follows from (B.1) that $F(A+h(A))=F(C)+G(C, X)+\hat{R}^{c}(X)$, where $\hat{R}^{c}(X)=F(X+\Delta h(X))+G(X+\Delta h(X), C)+G(C, \Delta h(X))$. On the other hand, using the definitions of $F$ and $G$, we find $F_{n}(C)=\alpha_{0}^{3 / 2} \delta_{n, 0}$, $G_{n}(C, X)=2 \alpha_{0} X_{n}+\alpha_{0} X_{-n}^{*}$. So, inserting $A=\hat{A}^{c}+X$ in (4.5) and noting that $\alpha_{0}(c) \hat{A}_{0}^{c} \equiv \alpha_{0}^{3 / 2}$, we obtain the following equation for $X$ :

$$
X_{n}^{\prime}=\alpha_{n}(c) X_{n}-v_{n}(c)\left(2 \alpha_{0} X_{n}+\alpha_{0} X_{-n}^{*}+\hat{R}_{n}^{c}(X)\right), \quad n \in \mathbf{Z} .
$$


Obviously, the linear operator in (C.17) is block diagonal. Choosing $X_{n}, X_{-}^{*}$ as (complex) variables, the $n^{\text {th }}$ block $(n \neq 0)$ is given by the $2 \times 2$ matrix:

$$
M_{n}^{c}=\left(\begin{array}{cc}
\alpha_{n}(c)-2 v_{n}(c) \alpha_{0} & -v_{n}(c) \alpha_{0} \\
-v_{-n}^{*}(c) \alpha_{0} & \alpha_{-n}^{*}(c)-2 v_{-n}^{*}(c) \alpha_{0}
\end{array}\right) .
$$

If $n=0$, we use the identity

$$
\begin{aligned}
& \alpha_{0}(c) X_{0}-v_{0}(c)\left(2 \alpha_{0} X_{0}+\alpha_{0} X_{0}^{*}\right) \\
& \quad=-\alpha_{0}\left(X_{0}+X_{0}^{*}\right)+\left(1-v_{0}(c)\right) \alpha_{0}\left(X_{0}+X_{0}^{*}\right)-v_{0}(c) \lambda_{0+}^{2} X_{0} \\
& \quad=-\alpha_{0}\left(X_{0}+X_{0}^{*}\right)+\tilde{R}^{c}(X),
\end{aligned}
$$

and include the quantity $\tilde{R}^{c}(X)$ in the non-linear term. Thus, choosing $\operatorname{Re} X_{0}$, $\operatorname{Im} X_{0}$ as (real) variables, the $0^{\text {th }}$ block is given by

$$
M_{0}^{c}=\left(\begin{array}{cc}
-2 \alpha_{0} & 0 \\
0 & 0
\end{array}\right) \text {. }
$$

So, defining $R_{n}^{c}(X)=-v_{n}(c) \hat{R}_{n}^{c}(X)+\delta_{n, 0} \tilde{R}^{c}(X)$, we can rewrite (C.17) in the form

$$
\begin{aligned}
\left(\begin{array}{c}
X_{n}^{\prime} \\
X_{*_{n}}^{* \prime}
\end{array}\right) & =M_{n}^{c}\left(\begin{array}{c}
X_{n} \\
X_{*_{n}}^{*}
\end{array}\right)+\left(\begin{array}{c}
R_{n}^{c}(X) \\
R_{-n}^{c *}(X)
\end{array}\right), \\
\left(\begin{array}{c}
\operatorname{Re} X_{0}^{\prime} \\
\operatorname{Im} X_{0}^{\prime}
\end{array}\right) & =M_{0}^{c}\left(\begin{array}{c}
\operatorname{Re} X_{0} \\
\operatorname{Im} X_{0}
\end{array}\right)+\left(\begin{array}{c}
\operatorname{Re} R_{0}^{c}(X) \\
\operatorname{Im} R_{0}^{c}(X)
\end{array}\right),
\end{aligned}
$$

which is nothing but (4.8).

Now, we bound the Lipschitz constant of the non-linear term $R^{c}(X)$ in the ball $\mathscr{B}_{2 \rho} \subset \mathscr{H}$. Since $\operatorname{Lip}(\Delta h) \leqq \frac{1}{2}\left(c_{0} / c\right)^{2}$ by Proposition 4.1, we see from (2.5), (4.3) that

$$
\begin{aligned}
\operatorname{Lip}\left(\hat{R}^{c}\right) & \leqq \operatorname{Lip}(F(\cdot+\Delta h(\cdot)))+\operatorname{Lip}(G(\cdot+\Delta h(\cdot), C))+\operatorname{Lip}(G(C, \Delta h(\cdot))) \\
& \leqq C \rho^{2}+C \rho+C\left(c_{0} / c\right)^{2} \leqq C\left(2 \rho+\left(c_{0} / c\right)^{2}\right),
\end{aligned}
$$

for some $C>0$, if $\rho \leqq 1$. On the other hand, it follows from (4.1), (4.3), (4.4) that $\left|v_{n}(c)\right|=\mathcal{O}(1)$ and $\operatorname{Lip}\left(\tilde{R}^{c}\right)=\mathcal{O}(1 / c)$ as $c \rightarrow \infty$. Thus, combining these results, we conclude that $\operatorname{Lip}\left(R^{c}\right) \leqq K_{5}\left(\rho+\left(c_{0} / c\right)\right)$ in $\mathscr{B}_{2 \rho} \subset \mathscr{H}$, for some $K_{5}>0$.

To complete the proof of Lemma 4.9, it remains to show that $\left\|e^{M_{n}^{c} t}\right\| \leqq \hat{D} e^{-\frac{1}{2} \alpha_{0} t}$ for all $t \in \mathbf{R}_{+}$and all $n \in \mathbf{Z}^{*}$, if $c$ is sufficiently large. Let $\mu_{n \pm}^{c}$ be the eigenvalues of the matrix $M_{n}^{c}$; we claim that $\operatorname{Re} \mu_{n \pm}^{c} \leqq-\frac{1}{2} \alpha_{0}$ for all $n$, if $c$ is sufficiently large. To see this, we first study the limiting matrix

$$
M_{n}=\lim _{c \rightarrow \infty} M_{n}^{c}=\left(\begin{array}{cc}
\alpha_{n}-2 \alpha_{0} & -\alpha_{0} \\
-\alpha_{0} & \alpha_{-n}-2 \alpha_{0}
\end{array}\right),
$$

whose eigenvalues are

$$
\mu_{n \pm}=\frac{1}{2}\left(\alpha_{n}+\alpha_{-n}-4 \alpha_{0} \pm \sqrt{4 \alpha_{0}^{2}+\left(\alpha_{n}-\alpha_{-n}\right)^{2}}\right) .
$$

Obviously, $\operatorname{Re} \mu_{n \pm} \leqq \max \left(\alpha_{n}, \alpha_{-n}\right)-\alpha_{0} \leqq \alpha_{1}-\alpha_{0}=\varepsilon^{2}-\alpha_{0}$ for all $|n|>1$. On the other hand, using (4.1), (4.4) and setting $\hat{q}_{n}=\max \left(\left|q_{n}\right|,\left|q_{-n}\right|\right)$, it is not difficult 
to show that $\left\|M_{n}^{c}-M_{n}\right\|=\mathcal{O}(1 / c)\left(1+\left|\hat{q}_{n}\right|\right)^{3}$ if $c \geqq 8\left(1+\left|\hat{q}_{n}\right|\right)$. It then follows from perturbation theory $[\mathrm{K}]$, that $\left|\mu_{n \pm}^{c}-\mu_{n \pm}\right| \leqq\left\|M_{n}^{c}-M_{n}\right\|$, if $\left\|M_{n}^{c}-M_{n}\right\|$ $\leqq \alpha_{0} / 2$.

Now, assume that $\left|\hat{q}_{n}\right| \leqq c^{1 / 4}$. Then $\left\|M_{n}^{c}-M_{n}\right\|=\mathcal{O}\left(c^{-1 / 4}\right) \leqq \alpha_{0} / 4$ if $c$ is sufficiently large, and thus $\operatorname{Re}\left(\mu_{n \pm}^{c}\right) \leqq \operatorname{Re}\left(\mu_{n \pm}\right)+\left|\mu_{n \pm}^{c}-\mu_{n \pm}\right| \leqq \varepsilon^{2}-\alpha_{0}+\alpha_{0} / 4$ $\leqq-\alpha_{0} / 2$. Conversely, if $\left|\hat{q}_{n}\right|>c^{1 / 4}$, we know from the proof of Lemma 4.7 that $\operatorname{Re} \alpha_{ \pm n}(c) \leqq-\sqrt{c} / 2$. Since the other matrix elements of $M_{n}^{c}$ are $\mathcal{O}(1)$ as $c \rightarrow \infty$, the same perturbation argument shows that $\mu_{n+}^{c}=\alpha_{n}(c)+\mathcal{O}(1)$ and $\mu_{n-}^{c}=$ $\alpha_{-n}^{*}(c)+\mathcal{O}(1)$. In particular, $\operatorname{Re} \mu_{n \pm}(c) \leqq-\alpha_{0} / 2$ if $c$ is sufficiently large.

To bound the exponential of $M_{n}^{c}$, we let $S_{n}^{c}$ be an invertible $2 \times 2$ matrix such that $\left(S_{n}^{c}\right)\left(M_{n}^{c}\right)\left(S_{n}^{c}\right)^{-1}$ is diagonal. Then $\left\|e^{M_{n}^{c} t}\right\| \leqq D_{n}^{c} e^{-\left(\alpha_{0} / 2\right) t}$ for all $t \in \mathbf{R}_{+}$, with $D_{n}^{c}=\left\|S_{n}^{c}\right\|\left\|\left(S_{n}^{c}\right)^{-1}\right\|$. It is not difficult to see that $\sup _{n} D_{n}^{c} \leqq \hat{D}<\infty$ for some $\hat{D}>1$, uniformly in $c$. The reason is that the quantities $\alpha_{n}(c), \alpha_{-}^{*}(c)$ in $M_{n}^{c}$ grow at least like $\sqrt{n}$ as $n \rightarrow \infty$, whereas the other matrix elements remain bounded; thus, $M_{n}^{c}$ is nearly diagonal if $n$ is large, and then $S_{n}^{c} \rightarrow 1$ as $n \rightarrow \infty$. The details of this argument are completed by separating the cases $\left|\hat{q}_{n}\right| \leqq c^{1 / 4},\left|\hat{q}_{n}\right|>c^{1 / 4}$.

Acknowledgements. We thank C.E. Wayne for a critical reading of the manuscript and for helpful discussions. This work has been supported by the Fonds National Suisse.

\section{References}

[A] Angenent, S.: private communication

[B] Ben-Jacob, E., Brand, H., Dee, G., Kramers, L., Langer, J.S.: Pattern propagation in non-linear dissipative systems. Physica 14D, 348-364 (1985)

[CE] Collet, P., Eckmann, J.-P.: Instabilities and Fronts in Extended Systems. Princeton, NJ: Princeton University Press 1990

[EW] Eckmann, J.-P., Wayne, C.E.: Propagating Fronts and the Center Manifold Theorem. Commun. Math. Phys. 136, 285-307 (1991)

[G] Gallay, Th.: A Center-Stable Manifold Theorem for Differential Equations in Banach Spaces. Commun. Math. Phys. 152, 249-268 (1993)

[K] Kato, T.: Perturbation Theory for Linear Operators. Berlin Heidelberg Springer New York: Springer 1990 